\title{
Dynamical Freezing and Scar Points in Strongly Driven Floquet Matter: Resonance vs Emergent Conservation Laws
}

\author{
Asmi Haldar $\odot,{ }^{1,3}$ Diptiman Sen $\odot,{ }^{2}$ Roderich Moessner, ${ }^{3}$ and Arnab Das ${ }^{1}$ \\ ${ }^{1}$ Indian Association for the Cultivation of Science, \\ $2 A \& 2 B$ Raja S. C. Mullick Road, Kolkata 700032, India \\ ${ }^{2}$ Centre for High Energy Physics and Department of Physics, Indian Institute of Science, \\ Bengaluru 560012, India \\ ${ }^{3}$ Max Planck Institute for the Physics of Complex Systems, Dresden, Germany
}

(Received 26 September 2019; revised 28 November 2020; accepted 12 February 2021; published 7 April 2021)

\begin{abstract}
We consider a clean quantum system subject to strong periodic driving. The existence of a dominant energy scale, $h_{D}^{x}$, can generate considerable structure in an effective description of a system that, in the absence of the drive, is nonintegrable and interacting, and does not host localization. In particular, we uncover points of freezing in the space of drive parameters (frequency and amplitude). At those points, the dynamics is severely constrained due to the emergence of an almost exact, local conserved quantity, which scars the entire Floquet spectrum by preventing the system from heating up ergodically, starting from any generic state, even though it delocalizes over an appropriate subspace. At large drive frequencies, where a naïve Magnus expansion would predict a vanishing effective (average) drive, we devise instead a strongdrive Magnus expansion in a moving frame. There, the emergent conservation law is reflected in the appearance of the "integrability" of an effective Hamiltonian. These results hold for a wide variety of Hamiltonians, including the Ising model in a transverse field in any dimension and for any form of Ising interaction. The phenomenon is also shown to be robust in the presence of two-body Heisenberg interactions with any arbitrary choice of couplings. Furthermore, we construct a real-time perturbation theory that captures resonance phenomena where the conservation breaks down, giving way to unbounded heating. This approach opens a window on the low-frequency regime where the Magnus expansion fails.
\end{abstract}

DOI: 10.1103/PhysRevX.11.021008

\section{INTRODUCTION}

For closed systems with time-independent Hamiltonians, the notion of ergodicity has been formulated at the level of eigenstates as the eigenstate thermalization hypothesis (ETH) $[1,2]$. According to the ETH, the expectation value of a local observable in a single energy eigenstate of a complex (disorder-free) many-body quantum system is equal to the thermal expectation value of the observable at a temperature corresponding to the energy density of that eigenstate. The implication of the ergodicity hypothesis in the context of time-dependent ("driven") closed quantum systems is an open question of fundamental importance.

Relatively recent progress along this line has occurred for systems subjected to a periodic drive (Floquet systems) $[3,4]$, which are perhaps conceptually closest to a static

Published by the American Physical Society under the terms of the Creative Commons Attribution 4.0 International license. Further distribution of this work must maintain attribution to the author(s) and the published article's title, journal citation, and DOI.
Subject Areas: Condensed Matter Physics, Quantum Physics, Statistical Physics system. These studies indicate that a quantum system that satisfies the ETH, when subjected to a periodic drive, approaches a state that locally looks like an entirely featureless "infinite-temperature" state. This case is in accordance with the ergodicity hypothesis-in systems that satisfy the ETH (we call them generic), energy is the only local conserved quantity, and any time dependence breaks this conservation, allowing the system to explore the entire Hilbert space.

The breakdown of the ETH in interacting systems due to the presence of localized states-either due to disorder (many-body localization) [5,6] or other mechanisms (like many-body Wannier-Stark localization) [7-9]—is well known within the equilibrium setup, and their persistence under periodic perturbations has also been observed [10-12]. Absolute stability bestowed upon such Floquet systems by disorder even allows for interesting spatiotemporal phases and long-range order in those systems [13,14]. But the common intuition is that a translationally invariant, interacting, nonintegrable, many-body system will be ergodic. However, this intuition has encountered a number of remarkable counterexamples recently within the static 
setting. It has been shown that in such systems, there can be highly excited energy eigenstates, dubbed scars, which do not satisfy the ETH [15-24]. Most of these examples (see, however, Ref. [25]) indicate the nontrivial (weak) breaking of ergodicity by certain eigenstates.

On the nonequilibrium side, stable Floquet states are seen in finite-size, closed, interacting Floquet systems, which are not localized in the absence of a drive [26-37]. In particular, it has recently been shown that ergodicity is broken in disorder-free generic systems under a periodic drive if the drive strength is greater than a threshold value (compared with the interaction strength) - a KAM-like scenario [38].

The emergence of constraints on dynamics and approximately (stroboscopically) conserved quantities under strong periodic driving is known for noninteracting systems: For strongly driven spin chains that can be mapped to free fermions, there exist special points in the space of the drive parameters, where any arbitrary initial state, for any (including infinite) system size, is frozen [39-43]. This situation is surprising since the appropriate description for such a system is a periodic generalized Gibbs' ensemble (PGE) [44]. Such an ensemble, though much less ergodic than a thermal one due to the presence of an extensive number of (periodically) conserved quantities, still leaves ample space for substantial dynamics. In particular, the emergent (approximate) conserved quantity is not one of the exact stroboscopically conserved quantities that characterizes the PGE, and hence, integrability does not necessarily assure its approximate conservation in any trivial way. Hence, in addition to the integrability, other constraints emerge at those special freezing points.

Here, we extend the reach of this phenomenology to generic interacting Floquet systems, far from integrability. Crucially, we also provide a physical mechanism and an analytical understanding of the resulting nonergodicity. Concretely, we demonstrate that generic, interacting, translationally invariant Ising systems as well as Heisenberg systems can exhibit nonergodic behavior under a strong periodic drive, and the nonergodicity is due to the emergence of a new approximate (stroboscopic) conservation law that is not present in the undriven quantum chaotic system. For certain isolated sets of values of the drive parameters - the scar or freezing points in the drive parameter space - the conservation is most accurate, leading to almost perfect freezing of the conserved quantity for any generic initial state.

The Floquet Hamiltonian is then no longer ergodic; i.e., its eigenstates (Floquet states) do not look like the otherwise expected infinite temperature states $[3,4]$ but instead are characterized by eigenvalues of the emergent conserved quantity because the dynamics does not mix different eigenstates with different eigenvalues of the emergent conserved quantity. However, this does not mean that there is no dynamics. Indeed, even at the scar points, we see pronounced dynamics evidenced by substantial growth in subsystem entanglement entropy as delocalization takes place within each eigenvalue sector. A finite-size analysis of the numerical results indicates the stability of the scars under an increase in the system size. This is quite distinct from emergence of nonthermal Floquet states only at finite sizes, where the trend towards thermalization is clearly visible with increasing system size [45].

We emphasize that-unlike the conventional scars, which are manifested as a few (measure zero) exceptional eigenstates-here, near the scar point, the Hilbert space is fractured into dynamically disjoint sectors, and hence the dynamics of any initial state is constrained.

At high driving frequencies, the conventional Magnus expansion-controlled by the driving frequency as the largest energy scale-fails, as the average Hamiltonian generally does not exhibit the conservation law in question. To remedy this, we present a strong-drive Magnus expansion, constructed in a "moving" frame incorporating the strong driving term. Here, the conservation law is manifest at low order in the expansion. For a general class of Hamiltonians, including the Ising model in a transverse field in any dimension and any form of the Ising interactions, we find that the effective Hamiltonian satisfies the conservation law up to the two leading orders for our example, capturing the freezing (observed from exact numerics) to a good approximation away from the resonances. The concordance of these results with exact numerics for finite size-systems suggest that the expansion is either convergent or asymptotic.

For lower drive frequencies, controlled approximation schemes for Floquet systems are sparse (see, however, Refs. [46-48]). Here, we formulate a novel perturbation theory, called Floquet-Dyson perturbation theory (FDPT), which again uses the fact that the drive amplitude is large. We find that this theory works best in the low-frequency regime, where we benchmark it for simple systems against an exact solution and against exact numerics. The advantage of our FDPT is that it enables us to account for isolated first-order resonances, which are of particular interest as their sparseness implies stable, nonthermal states at this order. The stability is maintained in the thermodynamic limit if our perturbation expansion is an asymptotic one, which is indicated by the finite-size analysis of our numerical results - the freezing is insensitive to an increase in the system size (see the finite-size results in Sec. VIC). In particular, the FDPT is remarkably accurate in predicting the resonances (obtained from exact numerics) close to integrability and hence at the scars. This provides a way to construct a stable Floquet state with the desired properties by choosing suitable drive terms.

We organize this paper as follows. After briefly introducing Floquet physics and our notations, we next present the phenomenology of scarring. We then develop the highfield Magnus expansion and the FDPT. Our presentation focuses on one particular quantum chaotic many-body 
model; we then demonstrate the key feature, namely, the emergence of the local conservation law and resulting absence of thermalization in other diverse models, that is, ones with three-spin interactions, long-range (power-law) interactions, and general Heisenberg interactions, establishing the generality of the nonergodic behavior that we have uncovered. We conclude with a summary and an outlook.

\section{FLOQUET IN A NUTSHELL}

The Floquet states $\left|\mu_{n}\right\rangle$ are elements of a complete orthonormal set of eigenstates of the time-evolution operator $U(T, 0)$ for time evolution from $t=0$ to $t=T$, for a system governed by a time-periodic Hamiltonian with a period $T=2 \pi / \omega$. The Floquet formalism is particularly useful for following the dynamics stroboscopically at discrete time instants $t=n T$. From the above definition, it follows that

$$
U(T, 0)\left|\mu_{n}\right\rangle=e^{-i \mu_{n}}\left|\mu_{n}\right\rangle,
$$

where the $\mu_{n}$ 's are real. It is customary to define an effective Floquet Hamiltonian $H_{\text {eff }}$ as

$$
U(T, 0)=e^{-i H_{\mathrm{eff}} T} .
$$

(We set $\hbar=1$ in this paper.) When observed stroboscopically at times $t=n T$, the dynamics can be thought of as being governed by the time-independent Hamiltonian $H_{\text {eff }}$, which has eigenvalues $\mu_{n} / T$ (modulo integer multiples of $2 \pi / T)$ and eigenvectors $\left|\mu_{n}\right\rangle$. In the infinite-time limit, the expectation values of a local operator $\mathcal{O}$ can be written in terms of the expectation values in the Floquet eigenstates as

$\lim _{N \rightarrow \infty}\langle\psi(N T)|\mathcal{O}| \psi(N T)\rangle=\sum_{n}\left|c_{n}\right|^{2}\left\langle\mu_{n}|\mathcal{O}| \mu_{n}\right\rangle=\mathcal{O}_{\mathrm{DE}}$,

where $|\psi(0)\rangle=\sum_{n} c_{n}\left|\mu_{n}\right\rangle$, and the subscript " $D E$ " denotes the diagonal ensemble average $[2,49-51]$ as defined above. The DE description is shown to be a very accurate description for generic interacting systems after a quench at long times [52]. A Floquet system under stroboscopic observation is equivalent to a quench with $H_{\text {eff }}$ in conjugation with stroboscopic observations. We mainly focus on the longitudinal magnetization (polarization in the $x$ direction), given by

$$
m^{x}=\frac{1}{L} \sum_{i}^{L} \sigma_{i}^{x}
$$

The diagonal ensemble average is equivalent to a "classical" average over the properties of the Floquet eigenstates $\left\{\left|\mu_{n}\right\rangle\right\}$. The diagonal ensemble average of $m^{x}$ is given by

$$
m_{\mathrm{DE}}^{x}=\sum_{n}\left|c_{n}\right|^{2}\left\langle\mu_{n}\left|m^{x}\right| \mu_{n}\right\rangle .
$$

The absence of interference between the Floquet states in a DE average ensures that it is sufficient to study the properties of individual Floquet states (and their spectrum average) in order to characterize the gross behavior of the driven system in the infinite-time limit. In the following, we therefore mostly concentrate on DE averages and the properties of the Floquet states.

\section{SCAR PHENOMENOLOGY}

\section{A. Freezing and emergent conservation}

In this section, we discuss the scar phenomenology for a periodically driven, interacting, nonintegrable Ising chain described by

$$
\begin{aligned}
H(t) & =H_{0}(t)+V, \quad \text { where } \\
H_{0}(t) & =H_{0}^{x}+\operatorname{Sgn}(\sin (\omega t)) H_{D}, \quad \text { with } \\
H_{0}^{x} & =-\sum_{n=1}^{L} J \sigma_{n}^{x} \sigma_{n+1}^{x}+\sum_{n=1}^{L} \kappa \sigma_{n}^{x} \sigma_{n+2}^{x}-h_{0}^{x} \sum_{n=1}^{L} \sigma_{n}^{x}, \\
H_{D} & =-h_{D}^{x} \sum_{n=1}^{L} \sigma_{n}^{x}, \quad \text { and } \\
V & =-h^{z} \sum_{n=1}^{L} \sigma_{n}^{z},
\end{aligned}
$$

where $\sigma_{n}^{x / y / z}$ are the Pauli matrices. Note that $H_{0}^{x}$ is, by definition, the sum of all the terms that commute with $H_{D}(t)$, and $V$ is the sum of all the remaining terms in the time-independent part of the Hamiltonian (while $V$ is called "perturbation" later, there is no implied distinction between the relative strengths of $H_{0}^{x}$ and $V$ ). This partition of the static part into $H_{0}^{x}$ and $V$ is for computational bookkeeping convenience for our analytical derivations.

The main result is that at large drive amplitude $h_{D}^{x}$, the longitudinal magnetization $m^{x}$ emerges as a emergent conserved quantity under the drive condition ("scar points" in the drive parameter space) given by

$$
h_{D}^{x}=k \omega,
$$

where $k$ are integers. Figure 1(a) shows that at the scar points (marked with arrows), the diagonal ensemble average $m_{\mathrm{DE}}^{x}$ [Eq. (3)] for $m^{x}$ is equal to the initial value $m^{x}(0)$, to very high accuracy, indicating that $m^{x}$ remains frozen at its initial value for arbitrarily long times. As seen from the figure, this happens for a very broad range of $\omega$.

However, as $h_{D}^{x}$ is reduced, the stable frozen regime eventually gives way to a Floquet thermalized regime. Below a clear freezing cutoff (around $h_{x}^{D} \approx 18$ ), $m_{\mathrm{DE}}^{x}$ 

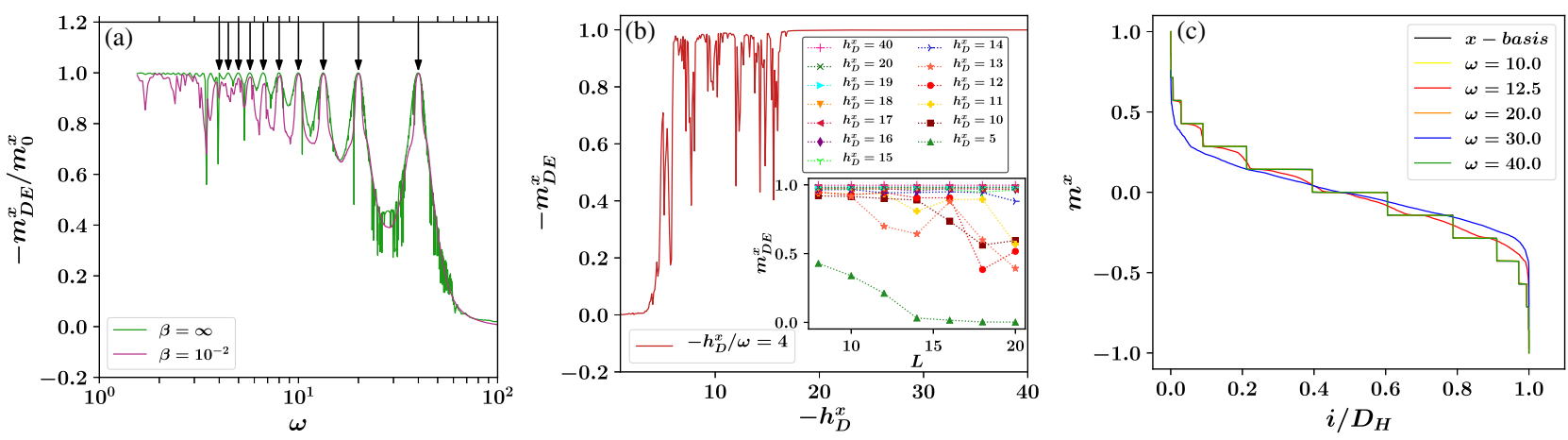

FIG. 1. Scars, resonances, and emergent conservation law. (a) $m_{\mathrm{DE}}^{x} / m_{0}^{x}$, the ratio of magnetizations after infinite (diagonal ensemble average) and 0 (initial state) cycles versus drive frequency $\omega$. Freezing, reflected in a large value of this ratio, occurs over a broad range of $\omega$ and is strongest at particular "scar" points (marked with arrows) $h_{D}^{x}=k \omega$, where $k$ is an integer (for $h_{D}^{x}=-40$ here, the ten arrows mark $\omega=40 / k ; k=1,2, \ldots, 10)$. Results are shown for zero and high-temperature initial states: the former being the ground state of $H(0)$ [which gives an initial magnetization $m^{x}(0) \lesssim 1$ ], and the latter being the Gibbs state with $\beta=10^{-2}\left[m^{x}(0) \approx 0.05\right]$ for $H_{I}$ of the form $H(0)$ but with $h_{D}^{x}=5$; all other parameters are the same as the driven Hamiltonian, namely, $J=1$, $\kappa=0.7 \pi / 3, h_{0}^{x}=e / 10, h_{D}^{x}=40, h^{z}=1.2, L=14$. The sharp dips in the green lines represent resonances, discussed in detail in the main text on Floquet-Dyson perturbation theory. Parameters are chosen to avoid these resonances. (b) $m^{x}$ as a function of $h_{D}^{x}$ for a fixed ratio $\left|h_{D}^{x} / \omega\right|=4$, showing the freezing cutoff $\left(h_{D}^{x} \approx 18\right)$ above which a stable regime of freezing sets in. Inset: finite-size behavior of the freezing. For intermediate strengths of the driving field, higher-order resonances lead to nonmonotonic behavior of $m_{\mathrm{DE}}^{x}$ on $L$, which is then sensitively dependent on small variations of $h_{D}^{x}$. (c) $\left\langle m^{x}\right\rangle$ of the Floquet states plotted against the serial number (normalized by the Hilbert space dimension $\left.D_{H}\right)$ of the Floquet states, arranged in decreasing order of $\left\langle m^{x}\right\rangle$. At the scar points $(\omega=10$, 20, and 40), the $\left\langle m^{x}\right\rangle$ values form steps coinciding with the eigenvalues of $m^{x}$ arranged and plotted in the same order: $m^{x}$ emerges as a emergent conserved quantity, hence the freezing of $m^{x}$ for any generic initial state.

exhibits strong fluctuations as a function of $h_{D}^{x}$ (short frozen stretches punctuated by higher-order resonance; see Sec. V B 1), followed by a subsequent sharp decline to almost zero below a thermalization threshold (around $h_{D}^{x} \approx 5$ ). A locally infinite-temperature-like Floquet thermalized regime is observed below this threshold, as shown in Fig. 1(b). This threshold does not exhibit any perceptible shift with system size [38]. The inset of Fig. 1(b) shows that there is no perceptible $L$ dependence in the freezing of $m_{\mathrm{DE}}^{x}$ as long as $h_{D}^{x}$ is above the freezing cutoff. The phenomenon is reminiscent of the nonmonotonic peak-valley structure of freezing observed in integrable Floquet systems in the thermodynamic limit $[39,40]$.

The figure shows that freezing happens for two very different kinds of initial states, namely, the highly polarized initial ground state of $H(0)$ as well as a high-temperature thermal state. The initial thermal density matrix is of the form

$$
\rho_{T h}(t=0)=\sum_{j=1}^{2^{L}} \frac{e^{-\beta \varepsilon_{j}}}{\mathcal{Z}}\left|\varepsilon_{j}\right\rangle\left\langle\varepsilon_{j}\right|,
$$

where $\left|\varepsilon_{j}\right\rangle$ is the $j$ th eigenstate of an initial Hamiltonian $H_{I}$, with eigenvalue $\varepsilon_{j}$. We have chosen $H_{I}=H(t=0$, $\left.h_{D}^{x}=5.0, h_{0}^{x}=0.1, J=1, \kappa=0.7\right)$, with $H(t)$ from Eq. (6), and $\mathcal{Z}=\sum_{j} e^{-\beta \varepsilon_{j}}$ is the partition function. Equation (8) represents a mixture of eigenstates $\left|\varepsilon_{j}\right\rangle$. Hence, we obtain the final diagonal ensemble density matrix by summing the diagonal ensemble density matrix for each $\left|\varepsilon_{j}\right\rangle$, weighted by its Boltzmann weight in $\rho_{T h}(0)$, i.e.,

$$
\begin{aligned}
\rho_{\mathrm{DE}}(t \rightarrow \infty) & =\sum_{j} \frac{e^{-\beta \varepsilon_{j}}}{\mathcal{Z}}\left(\sum_{k}\left|\left\langle\varepsilon_{j} \mid \mu_{k}\right\rangle\right|^{2}\left|\mu_{k}\right\rangle\left\langle\mu_{k}\right|\right) \\
& =\sum_{k}\left(\sum_{j} \frac{e^{-\beta \varepsilon_{j}}}{\mathcal{Z}}\left|\left\langle\varepsilon_{j} \mid \mu_{k}\right\rangle\right|^{2}\right)\left|\mu_{k}\right\rangle\left\langle\mu_{k}\right| .
\end{aligned}
$$

The emergent conservation of $m^{x}$ for a generic thermal state suggests that all the Floquet states must be organized according to the conservation law. This case is shown to be true in Fig. 1(c), which displays the expectation value $\left\langle m^{x}\right\rangle$ in the Floquet eigenstates [corresponding to the drive in Fig. 1(c)], plotted against their serial number (normalized by the dimension $D_{H}$ of the Hilbert space), arranged in decreasing order of their $\left\langle m^{x}\right\rangle$ values. For the scar points, for $h_{D}^{x}=40$ at $\omega=10,20,40$, the values of $\left\langle m^{x}\right\rangle$ of the Floquet states coincide with the eigenvalues of $m^{x}$, indicating that all the eigenstates of $m^{x}$ that participate in a given Floquet state have the same $m^{x}$ eigenvalues. This result explains the conservation or freezing of $m^{x}$ for dynamics starting with any generic initial state. As we will see later, the condition for encountering such a scar point [Eq. (7)] can be deduced from both the FDPT and a Magnus expansion in a timedependent frame, and the latter confirms the effect over the entire spectrum and explains the steps in $\left\langle m^{x}\right\rangle$ to the leading orders. 


\section{B. Dynamics of the unentangled eigenstates of $m^{x}$ : Growth of entanglement entropy}

We define an unentangled, complete, orthonormal set of eigenstates of $m^{x}$, which we call the $x$ basis. Each element of the $x$ basis is a simultaneous eigenstate of all the $\sigma_{i}^{x}$ operators. The nontriviality of the dynamics at the scar points and the consequence of the emergent conservation are manifested in the growth of the half-chain entanglement entropy $E_{\frac{1}{2}}$ at the scar points, especially with different $x$ basis eigenstates of $m^{x}$ as initial states. We study the halfchain entanglement entropy

$$
E_{\frac{1}{2}}=-\operatorname{Tr}\left[\rho_{\frac{1}{2}} \log _{2} \rho_{\frac{1}{2}}\right]
$$

where $\rho_{\frac{1}{2}}$ is the density matrix of one half of the chain, obtained by tracing out the other half.

The results are shown in Fig. 2. These results highlight that, even though $m^{x}$ is conserved for large enough $h_{D}^{x}$ at the scar points, there is substantial dynamics even at those points. For large enough $h_{D}^{x}$, Figs. 2(d)-2(i), we see that different eigenstates of $m^{x}$ evolve quite differently even at the scar points, at which $m^{x}$ is conserved to a very good approximation for all initial states. For example, for the fully polarized initial state, entanglement does not grow even after $10^{10}$ drive cycles, but for the Néel and the $L / 2$ domain-pair initial states, it does. This reflects the respective sizes of the $m^{x}$ subspaces with maximal and zero magnetization.

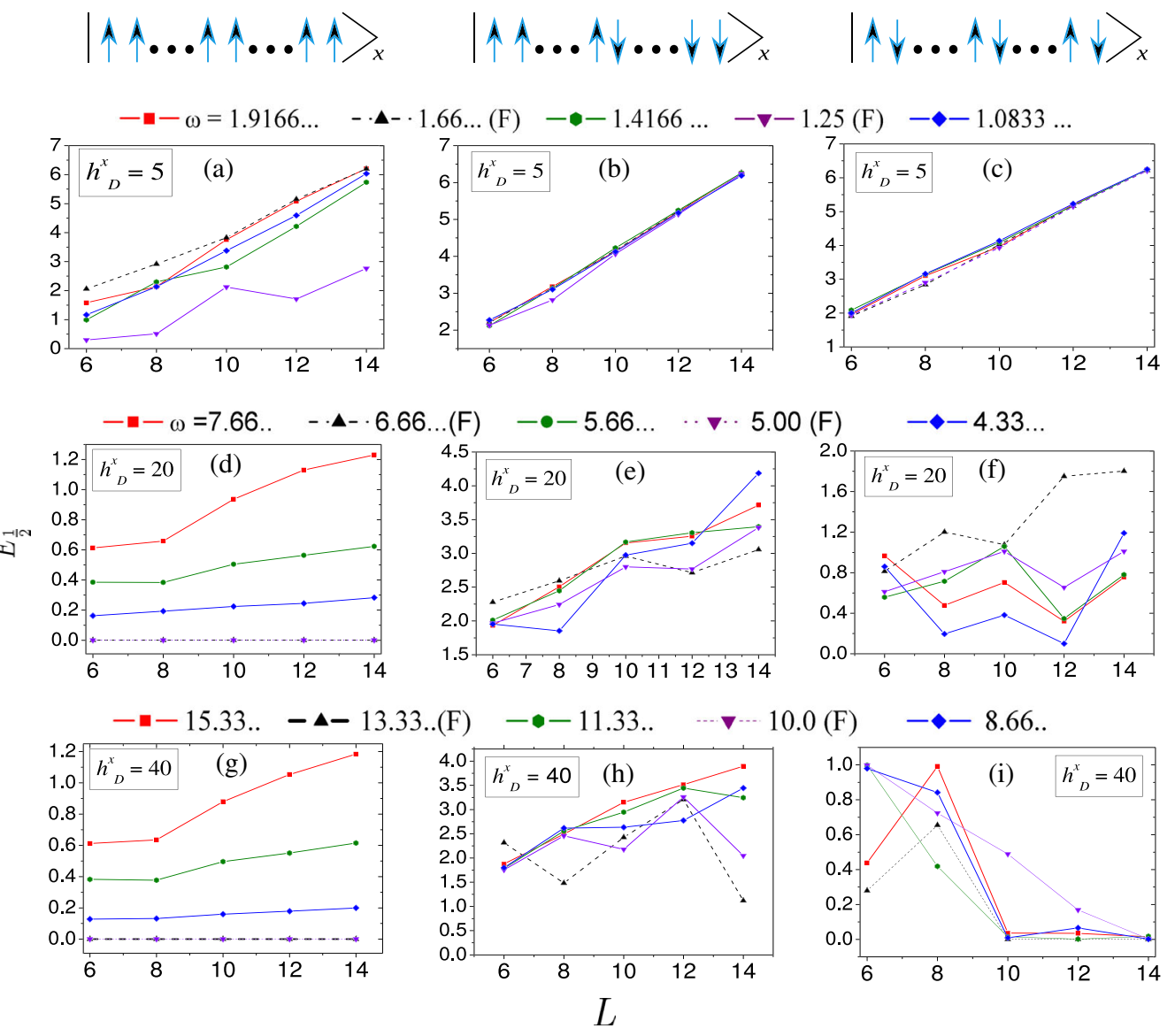

FIG. 2. (De)localization of the wave function over the $x$ basis (simultaneous eigenstates of all of the $\sigma_{i}^{x}$ 's) as evidenced by the halfchain entanglement entropy $\left(E_{\frac{1}{2}}\right)$ versus system size $L$, for different driving strengths $h_{D}^{x}$ (rows) and initial states (left column: maximally $m^{x}$ polarized; middle: $L / 2$-domain-pair state with vanishing total $m^{x}$; right: Néel state). Top row (small $h_{D}^{x}=5$ ): $E_{\frac{1}{2}}$ entropy grows linearly with system size for all initial states, signaling ergodicity. For stronger drives $\left(h_{D}^{x}=20\right.$ and 40 in the middle and bottom rows, respectively), scars appear, and $E_{\frac{1}{2}}$ depends strongly on the initial states, reflecting the size of the emergent magnetization sectors: For the fully polarized initial states (left column), $E_{\frac{1}{2}}$ does not grow at all for the freezing or scar points [marked as (F) in the figure legends and represented by almost indistinguishably coincidental black and violet triangles], while for the Néel and the $L / 2$-domain-pair initial states, there is considerable growth in $E_{\frac{1}{2}}$ even at the scar points, reflecting (at least partial) delocalization over the large concomitant magnetization sectors. The results are for $J=1, \kappa=0.7, h_{0}^{x}=e / 10, h^{z}=1.2, L=14$, averaged over $10^{4}$ cycles after driving for $10^{10}$ cycles. 
The growth of $E_{\frac{1}{2}}$ also reflects the role of interactions in the dynamics even at the scar points, without which we would not see such a substantial growth of entanglement.

In the Appendix $\mathrm{C}$, we show that the suppression of entanglement growth is robust in that it is observed for other patterns of the drive field, as long as the concomitant emergent conservation law gives rise to well-defined sectors that contain only a small number of states.

\section{STRONG-DRIVE MAGNUS EXPANSION}

We next provide a modified Magnus expansion that incorporates the large size of the drive from the start, using the inverse of the driving field as a small parameter. This approach makes the emergence of a conserved quantity manifest, for a wide range of Hamiltonians - the terms in the time-independent part of the Hamiltonian that commute with the time-dependent part of the Hamiltonian $\left(H_{0}^{x}\right.$ here) can have any form because the factor premultiplying the terms involving $H_{0}^{x}$ vanishes to second order regardless of the form of $H_{0}^{x}$. For example, it applies to transverse-field Ising models in any dimension, with any Ising interaction. Using this approach, one can immediately read off the scars found above.

The conventional Magnus expansion uses the inverse of a large frequency as a small parameter (see, e.g., Refs. [28,53]) for obtaining the Floquet Hamiltonian $H_{\text {eff }}$ [Eq. (2)] as given below.

$$
\begin{aligned}
& H_{\mathrm{eff}}=\sum_{n=0}^{\infty} H_{F}^{(n)}, \quad \text { where } \\
& H_{F}^{(0)}=\frac{1}{T} \int_{0}^{T} d t H(t), \\
& H_{F}^{(1)}=\frac{1}{2 !(i) T} \int_{0}^{T} d t_{1} \int_{0}^{t_{1}} d t_{2}\left[H\left(t_{1}\right), H\left(t_{2}\right)\right],
\end{aligned}
$$

and so on. In our case, we have $h_{D}^{x}>\omega$, making the series nonconvergent even when $\omega$ is greater than all other couplings in the Hamiltonian, so the naïve Magnus expansion is qualitatively wrong even at leading order: The first-order term $H^{(0)}$ is the time average over one period of $H(t)$ [Eq. (6)], an interacting, generic Hamiltonian that does not conserve $m^{x}$. Hence, we would have no hint of the scars even from the first-order term.

This problem can be remedied when the strong drive modulates the strength of a fixed field or potential (this is a very natural way of applying a periodic drive). The largest coupling ( $h_{D}^{x}$ here) can be eliminated from the Hamiltonian by switching to a time-dependent frame as follows [28]. We introduce a unitary transformation

$$
\begin{aligned}
\left|\psi_{\text {mov }}(t)\right\rangle & =W(t)^{\dagger}|\psi(t)\rangle, \\
\hat{\mathcal{O}}_{\text {mov }} & =W(t)^{\dagger} \hat{\mathcal{O} W} W(t),
\end{aligned}
$$

where $|\psi(t)\rangle$ is the wave function and $\hat{\mathcal{O}}$ is any predefined operator (the subscript "mov" marks the quantities in the moving frame).

The crux of the expansion is then apparent for a $W(t)$ of the following form,

$$
W(t)=\exp \left[-i \int_{0}^{t} d t^{\prime} r\left(t^{\prime}\right) H_{D}\right],
$$

where $r(t)$ is a $T$-periodic parameter. If the total Hamiltonian were constant up to the time-dependent prefactor $r(t)$, i.e., $H(t)=r(t) H(0)$, the above would just give the solution of the static Schrödinger equation, but with a rate of phase accumulation for each (timeindependent) eigenstate given by the integrand of the variable prefactor. In particular, any conservation law of $H(0)$ would be bequeathed to the time-dependent problem. Now, if the drive is not the only, but still the dominant part of the Hamiltonian, there will be corrections to this picture; however, this suggests the eigenbasis of the drive and its conservation law(s) should remain perturbatively useful starting points.

Given the form of $H_{D}(t)$ in Eq. (6), the transformed Hamiltonian reads

$$
H_{\mathrm{mov}}=W(t)^{\dagger} H(t) W(t)-i W(t)^{\dagger} \partial_{t} W,
$$

where the second term exactly cancels the part from the first term that has $h_{D}^{x}$ as its coupling; hence, $H_{\text {mov }}$ is free from any coupling of order $h_{D}^{x}$ (see Appendix A 3 for details).

\section{A. Scars in the driven interacting Ising chain}

In the case of Eq. (6), we have

$$
H(t)=H_{0}^{x}+V-\operatorname{Sgn}(\sin (\omega t)) h_{D}^{x} \sum_{i} \sigma_{i}^{x} .
$$

Switching to the moving frame by using the transformation in Eq. (13) gives

$$
\begin{aligned}
H_{\text {mov }} & =H_{0}^{x}-h^{z} \sum_{i}\left[\cos (2 \theta) \sigma_{i}^{z}+\sin (2 \theta) \sigma_{i}^{y}\right], \quad \text { where } \\
\theta(t) & =-h_{D}^{x} \int_{0}^{t} d t^{\prime} \operatorname{Sgn}\left(\sin \omega t^{\prime}\right) .
\end{aligned}
$$

After some algebra, we find the Magnus expansion of $H_{\text {mov }}$ to have the following leading terms:

$$
\begin{aligned}
H_{F}^{(0)}= & H_{0}^{x}-\frac{h^{z}}{h_{D}^{x} T}\left[\sin \left(h_{D}^{x} T\right) \sum_{i} \sigma_{i}^{z}\right. \\
& \left.-\left(1-\cos \left(h_{D}^{x} T\right)\right) \sum_{i} \sigma_{i}^{y}\right] .
\end{aligned}
$$


Note that this is useful for $h_{D}^{x} \gg 1 / T$, the regime we are interested in. The next-order term is given by

$$
H_{F}^{(1)}=\frac{1}{2 ! T i} \int_{0}^{T} d t_{1} \int_{0}^{t_{1}} d t_{2}\left[H^{\mathrm{mov}}\left(t_{1}\right), H^{\mathrm{mov}}\left(t_{2}\right)\right] .
$$

Denoting $\theta\left(t_{1}\right)=\theta_{1}, \theta\left(t_{2}\right)=\theta_{2}, \sum_{i} \sigma_{i}^{z / y}=S^{z / y}$, and the form of $H_{\text {mov }}$ from Eq. (16), we get

$$
\begin{aligned}
H_{F}^{(1)}= & {\left[S^{z}, H_{0}^{x}\right] h^{z}\left(\cos 2 \theta_{2}-\cos 2 \theta_{1}\right) } \\
& +\left[S^{y}, H_{0}^{x}\right] h^{z}\left(\sin 2 \theta_{2}-\sin 2 \theta_{1}\right) \\
& +\left[S^{y}, S^{z}\right]\left(h^{z}\right)^{2} \sin \left(2 \theta_{1}-2 \theta_{2}\right) .
\end{aligned}
$$

Upon integration (see Appendix A 1 and A 3 for details), this identically gives

$$
H_{F}^{(1)}=0
$$

The end result-a homogeneous expansion in the small parameters $1 / h_{D}^{x}$ and $1 / T$ from the two initial ordersgiven in Eq. (17), is quite remarkable. First, for $h_{D}^{x} T=2 \pi k$ (where $k$ can be any integer), $H_{F}^{(0)}=H_{0}^{x}$; this is precisely the condition for scars observed numerically [Eq. (7)] and also from the FDPT [see Eq. (44)]. Clearly, to this approximation, $H_{\text {eff }}$ not only has a conservation law but is also integrable; indeed, it is classical, with all terms commuting. Numerical results suggest that the above expansion (unlike the Magnus expansion in the static frame) is an asymptotic one, at least in the neighborhood of the scar points, since the leading-order terms represent the exact numerical results accurately.

Second, it is clear from the forms of $H_{F}^{(0)}$ and $H_{F}^{(1)}$ that the results hold independently of the form of $H_{0}^{x}$; this could be in any spatial dimension and can incorporate any form of Ising interactions. This wide generality implies that stable emergent conservation laws and constraints (in keeping with the possible asymptotic nature of the expansion) may emerge in generic interacting Floquet systems in the thermodynamic limit. Since $H_{x}^{0}$ is, by definition, the portion of the static part of the Hamiltonian that commutes with $H_{D}$, the statement of generality obtained from the above analysis stands as follows: While the nature of the whole static part can be tuned over a wide variety of many-body Hamiltonians depending on the form of $H_{0}^{x}$ (ranging from noninteracting to interacting, integrable to nonintegrable, low to high dimensional), the emergence of the conservation law and the resultant scarring do not depend on the form of $H_{0}^{x}$. In Sec. VI, we support this statement by considering various kinds of Ising interactions, and going beyond, we demonstrate the freezing in the presence of anisotropic Heisenberg interactions.

\section{FLOQUET-DYSON PERTURBATION THEORY}

In this section, we develop a theory that opens a window on the otherwise difficult-to-access [46-48] lowfrequency regime. We first test it for an exactly soluble problem and then apply it to the Ising chain studied in the previous section.

We find that the theory provides valuable insights for both systems. In particular, it identifies a resonance condition corresponding to the dips, as well as a freezing condition corresponding to the maxima in the response plotted in Figs. 4 and 1, respectively. A concurrence of the two accounts for the varying dip depths in that figure. While a comprehensive treatment of the general manybody problem is not yet possible, we believe that these items capture ingredients central for its understanding.

We first present the general formulation of the FDPT. The goal is to construct the Floquet states $\left|\mu_{n}\right\rangle$. We resort to a setting where the unperturbed Hamiltonian is time dependent and the perturbation is static [54]. The central idea is to construct the Floquet states in the presence of the small static perturbation from the known unperturbed Floquet states by applying time-dependent perturbation theory (a Dyson-like series for the wave function). For this process, one needs to know the unperturbed Floquet states, which come from the solution of the time-dependent Schrödinger equation with only the time-dependent part in the Hamiltonian (including the static parts that commute with it at all times). In our case, this is naturally achieved as follows. The central ingredient is that the driven Hamiltonian

$$
H(t)=H_{0}(t)+V
$$

contains a large time-dependent term $H_{0}(t)$, which has a time-independent set of eigenstates and a perturbation $V$ that is time independent. Those states then serve as the unperturbed Floquet states, and $V$ can then be treated as a small (compared to the drive amplitude) perturbation.

We work in the basis of eigenstates of $H_{0}(t)$ (these are the unperturbed Floquet states), denoted as $|n\rangle$, so that

$$
H_{0}(t)|n\rangle=E_{n}(t)|n\rangle,
$$

and $\langle m \mid n\rangle=\delta_{m n}$.

Next, we assume, without loss of generality, that $V$ is completely off-diagonal in this basis, namely,

$$
\langle n|V| n\rangle=0
$$

for all $n$. We now find solutions of the time-dependent Schrödinger equation

$$
i \frac{\partial\left|\psi_{n}\right\rangle}{\partial t}=H(t)\left|\psi_{n}(t)\right\rangle,
$$


which satisfy

$$
\left|\psi_{n}(T)\right\rangle=e^{-i \mu_{n}}\left|\psi_{n}(0)\right\rangle .
$$

For $V=0$, each eigenstate $|n\rangle$ of $H_{0}(t)$ is a Floquet state, with Floquet quasienergy $\mu_{n}^{(0)}=\int_{0}^{T} d t E_{n}(t)$ (defined modulo $2 \pi$ ).

For $V$ nonzero but small, we develop a Dyson-like series for the wave function to first order in $V$. Clearly, $V$ is a small perturbation as long as $\left|V / h_{D}^{x}\right| \ll 1$, though it can otherwise be comparable to or larger than the other couplings of the undriven Hamiltonian. In our ansatz, the $n$th eigenstate is written as

$$
\left|\psi_{n}(t)\right\rangle=\sum_{m} c_{m}(t) e^{-i \int_{0}^{t} d t^{\prime} E_{m}\left(t^{\prime}\right)}|m\rangle,
$$

where $c_{n}(t) \simeq 1$ for all $t$ while $c_{m}(t)$ is of order $V$ (and therefore small) for all $m \neq n$ and all $t$.

We then substitute the form for the wave function in Eq. (26) in the time-dependent Schrödinger equation and then apply the key condition of the method; namely, we demand $\left|\psi_{n}(0)\right\rangle=\left|\mu_{n}\right\rangle$, i.e.,

$$
\left|\psi_{n}(T)\right\rangle=e^{i \mu_{n}}\left|\psi_{n}(0)\right\rangle .
$$

Then, taking the overlaps with the basis states $|m\rangle$, we find (for details of the algebra, see Appendix B)

$$
c_{m}(0)=-i\langle m|V| n\rangle \frac{\int_{0}^{T} d t e^{i \int_{0}^{t} d t^{\prime}\left[E_{m}\left(t^{\prime}\right)-E_{n}\left(t^{\prime}\right)\right]}}{e^{i \int_{0}^{T} d t\left[E_{m}(t)-E_{n}(t)\right]}-1} .
$$

We see that $c_{m}(t)$ is indeed of order $V$ provided that the denominator on the right-hand side of Eq. (28) does not vanish; we call this case nondegenerate. If

$$
e^{i \int_{0}^{T} d t\left[E_{m}(t)-E_{n}(t)\right]}=1,
$$

we have a resonance between states $|m\rangle$ and $|n\rangle$, and the above analysis breaks down. Now, if there are several states that are connected to $|n\rangle$ by the perturbation $V$, Eq. (28) describes the amplitude to go to each of them from $|n\rangle$. Up to order $V^{2}$, the total probability of excitation away from $|n\rangle$ is given by $\sum_{m \neq n}\left|c_{m}(0)\right|^{2}$ at time $t=0$.

\section{A. Single large spin: An exactly soluble test bed}

As a simple illustration of the FDPT, we discuss a system with a single spin governed by a time-dependent Hamiltonian. We briefly discuss some results obtained from the FDPT (which give the conditions for perfect freezing and resonances), numerical results, and exact results for the Floquet operator. The details are presented in Appendix B.

\section{Model}

We consider a single spin $\vec{S}$, with $\vec{S}^{2}=S(S+1)$, which is governed by a Hamiltonian of the form

$$
H(t)=-h^{x} S^{x}-h^{z} S^{z}-h_{D}^{x} \operatorname{Sgn}(\sin (\omega t)) S^{x} .
$$

The time period is $T=2 \pi / \omega$. Since $\sin (\omega t)$ is positive for $0<t<T / 2$ and negative for $T / 2<t<T$, the Floquet operator is given by

$$
U=e^{(i T / 2)\left[\left(h^{x}-h_{D}^{x}\right) S^{x}+h^{z} S^{z}\right]} \times e^{(i T / 2)\left[\left(h^{x}+h_{D}^{x}\right) S^{x}+h^{z} S^{z}\right]} .
$$

It is clear from the group properties of matrices of the form $e^{i \vec{a} \cdot \vec{S}}$ that $U$ in Eq. (31) must be of the same form and can be written as

$$
\begin{aligned}
U & =e^{i \gamma \hat{k} \cdot \vec{S}}, \\
\text { where } \hat{k} & =(\cos \theta, \sin \theta \cos \phi, \sin \theta \sin \phi) .
\end{aligned}
$$

We work in the basis in which $S^{x}$ is diagonal. Since the eigenstates of $U$ in Eq. (32) are the same as the eigenstates of the matrix $M=\hat{k} \cdot \vec{S}$, the expectation values of $S^{x}$ in the different eigenstates take the values $\cos \theta$ times $S, S-1, \ldots,-S$. The maximum expectation value is given by $m_{\max }^{x}=S \cos \theta$.

\section{Analytical results from FDPT}

We can use the FDPT to derive the correction to $m_{\max }^{x}$ to first order in the small parameter $h^{z} / h_{D}^{x}$. Namely, we find how the state given by $|0\rangle \equiv\left|S^{x}=S\right\rangle$ mixes with the state $|1\rangle \equiv\left|S^{x}=S-1\right\rangle$. We discover that

$$
c_{1}(0)=\frac{\sqrt{2 S} h^{z}}{h_{D}^{x}} \frac{e^{i h^{x} T / 2}\left[e^{i h_{D}^{x} T / 2}-\cos \left(h^{x} T / 2\right)\right]}{e^{i h^{x} T}-1} .
$$

Three possibilities arise at this stage.

(i) The denominator of Eq. (33) is not zero. Then, the expectation value of $S^{x}$ in this state will be close to $S$ since $h^{z} / h_{D}^{x}$ is small. In addition, if the numerator of Eq. (33) vanishes, we get perfect freezing, namely, $\left\langle S^{x}\right\rangle=S$.

(ii) The denominator of Eq. (33) vanishes; i.e., $h^{x}$ is an integer multiple of $2 \pi / T$, but the numerator does not vanish. This is called the resonance condition. Clearly, the perturbative result for $c_{1}(0)$ breaks down in this case, and we have to either develop a degenerate perturbation theory or do an exact calculation.

(iii) Both the numerator and the denominator of Eq. (33) vanish. Once again, the perturbative result breaks down, and we have to do a more careful calculation.

Here, we comment on the dependence of the result in Eq. (33) on the value of $S$. At $t=0$, the probability of state 
$|1\rangle$ is $\left|c_{1}(0)\right|^{2}$, and the probability of state $|0\rangle$ is $1-\left|c_{1}(0)\right|^{2}$. Hence, the expectation value of $S^{x} / S$ is given by

$$
\begin{aligned}
\frac{m_{\max }^{x}}{S}= & \frac{1}{S}\left[S\left(1-\left|c_{1}(0)\right|^{2}\right)+(S-1)\left|c_{1}(0)\right|^{2}\right] \\
= & 1-2\left(\frac{h^{z}}{h_{D}^{x}}\right)^{2} \\
& \times \frac{1+\cos ^{2}\left(h^{x} T / 2\right)-2 \cos \left(h^{x} T / 2\right) \cos \left(h_{D}^{x} T / 2\right)}{4 \sin ^{2}\left(h^{x} T / 2\right)} .
\end{aligned}
$$

We expect Eq. (33) to break down at a sufficiently large value of $S$ since it was derived using first-order perturbation theory, which is accurate only if $\left|c_{1}(0)\right| \ll 1$. However, we observe that the value of $m_{\max }^{x} / S$ in Eq. (34) is independent of $S$. Therefore, in this model, we have the striking result that we can use first-order perturbation theory for values of $S$ that are not large to derive an expression like Eq. (34), which is then found to hold for arbitrarily large values of $S$.

\section{Numerical results}

Given the values of the parameters $S, T, h^{x}, h^{z}$, and $h_{D}^{x}$, we can numerically compute $U$ and its eigenstates. From the eigenstates, we can calculate $m_{\max }^{x}$, which is the maximum value of the expectation value of $\left\langle S^{x}\right\rangle$. In Fig. 3, we plot $m_{\max }^{x}$ versus $h^{x}$, for $S=20, T=10$, $h^{z}=1$, and (a) $h_{D}^{x}=40$ and (b) $h_{D}^{x}=12.8 \pi \simeq 40.212$. In Fig. 3(a), we see large dips for $h^{x}$ equal to all integer multiples of $2 \pi / T$. In Fig. 3(b), we see large dips for $h^{x}$ equal to odd integer multiples of $2 \pi / T$, but the dips are much smaller for $h^{x}$ equal to even integer multiples of $2 \pi / T$.

We can understand these results using the FDPT. In Fig. 3(a), we have $h_{D}^{x}=40$; hence, $\cos \left(h_{D}^{x} T / 2\right) \neq \pm 1$, and the numerator of Eq. (33) can never vanish. We therefore obtain large dips for $h^{x}$ equal to all integer multiples of $2 \pi / T$, where the denominator of Eq. (33) vanishes
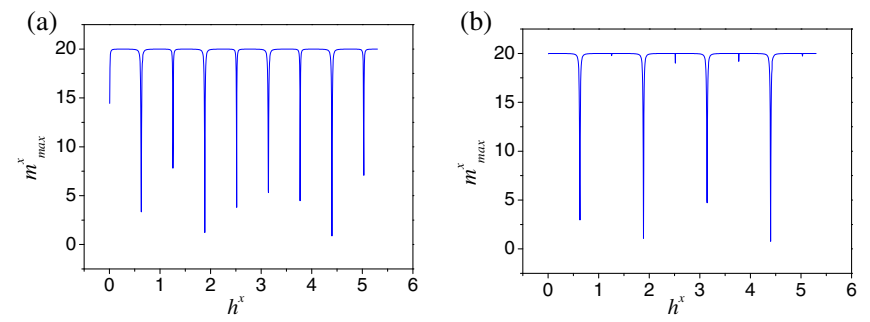

FIG. 3. Plots of the maximum expectation value of $S^{x}$ versus $h^{x}$, for $S=20, T=10, h^{z}=1$, and (a) $h_{D}^{x}=40$ and (b) $h_{D}^{x}=$ $12.8 \pi \simeq 40.212$. In panel (a), we see pronounced dips for $h^{x}$ equal to all integer multiples of $2 \pi / T$, while in panel (b) we see pronounced dips only when $h^{x}$ is equal to odd integer multiples of $2 \pi / T$, as predicted by the FDPT result, Eq. (33). [case (ii)]. However, in Fig. 3(b), $h_{D}^{x}=12.8 \pi$, so $\cos \left(h_{D}^{x} T / 2\right)=1$. Hence, both the numerator and the denominator of Eq. (33) vanish when $h^{x}$ is equal to even integer multiples of $2 \pi / T$ [case (iii)]. This explains why the dips in $m_{\max }^{x}$ are much smaller for $h^{x}$ equal to even integer multiples of $2 \pi / T$, but they continue to be large for $h^{x}$ equal to odd integer multiples of $2 \pi / T$.

\section{Form of the Floquet operator in different cases}

We now present expressions for the Floquet operator $U$ in Eq. (32) based on the exact results derived in Appendix B 1 a. The purpose of this exercise is to show that the form of $U$ is quite different in cases (i)-(iii).

Assuming that $h_{D}^{x}$ is positive and much larger than $\left|h^{x}\right|$ and $\left|h^{z}\right|$, we find, to zeroth order in $h^{z} / h_{D}^{x}$, that

$$
\cos \left(\frac{\gamma}{2}\right)=\cos \left(\frac{h^{x} T}{2}\right) \quad \text { and } \quad \hat{k}=\hat{x}
$$

provided that $e^{i h^{x} T} \neq 1$ [case (i)]. Equation (35) implies that the Floquet operator corresponds to a rotation about the $\hat{x}$ axis by an angle $\gamma$.

If $e^{i h^{x} T}=1$, i.e., $\cos \left(h^{x} T / 2\right)= \pm 1$, but $\cos \left(h^{x} T / 2\right) \neq$ $e^{i h_{D}^{x} T / 2}$, the denominator of Eq. (33) vanishes but the numerator does not [case (ii), called the resonance condition]. It turns out that we then have to expand up to second order in $h^{z} / h_{D}^{x}$, which gives

$$
\begin{aligned}
\hat{k}= & \cos \left(\frac{h_{D}^{x} T}{4}\right) \hat{z}-\sin \left(\frac{h_{D}^{x} T}{4}\right) \hat{y} \\
& \text { if } \cos \left(\frac{h^{x} T}{2}\right)=1, \\
= & \sin \left(\frac{h_{D}^{x} T}{4}\right) \hat{z}+\cos \left(\frac{h_{D}^{x} T}{4}\right) \hat{y} \\
& \text { if } \cos \left(\frac{h^{x} T}{2}\right)=-1 .
\end{aligned}
$$

This implies that the Floquet operator corresponds to a rotation about an axis lying in the $y-z$ plane. Thus, the expectation value of $S^{x}$ will be zero in all the eigenstates of the Floquet operator.

Finally, if $e^{i h^{x} T}=1$ and $\cos \left(h^{x} T / 2\right)=e^{i h_{D}^{x} T / 2}$, both the numerator and the denominator of Eq. (33) vanish [case (iii)]. We then discover that

$$
\hat{k}=\frac{h^{x} \hat{x}-h^{z} \hat{z}}{\sqrt{\left(h^{z}\right)^{2}+\left(h^{x}\right)^{2}}} .
$$

Hence, the Floquet operator corresponds to a rotation about an axis lying in the $x-z$ plane.

To summarize, assuming that $h^{z} / h_{D}^{x}$ is small, we obtain quite different results depending on which of the three cases (i)-(iii) arise. We see these differences both in the 
numerical results for $m_{\max }^{x}$ shown in Fig. 3 and in the forms of the Floquet operator in Eqs. (35)-(37), which are obtained by an exact calculation.

\section{B. FDPT for the interacting Ising chain}

Now, we apply FDPT to our interacting Ising chain [Eq. (6)], studied numerically above. We set $h^{z} \ll h_{D}^{x}$, and treat $V$ as the perturbation. We use periodic boundary conditions.

The eigenstates $|n\rangle$ of $H_{0}(t)$ are diagonal in the basis of the operators $\sigma_{n}^{x}$. In particular, the state in which all spins $\sigma_{n}^{x}=+1$ will be denoted as $|0\rangle$, and we start by calculating the Floquet state $\left|m_{\max }^{x}\right\rangle$ (maximally polarized Floquet state) obtained by perturbing this state to first order in $h^{z} / h_{D}^{x}$. While calculating $m^{x}$ from perturbation theory, we use this Floquet state.

The rationale for this is as follows. First, if we start with a fully polarized state in the $+x$ direction (as is done, for example, in the experiments by Monroe [55]) or with the ground state of $H(0)$, with $h_{D}^{x} \gg h^{z}, \kappa$, then the initial state is expected to have a strong overlap with this particular Floquet state. Hence, at very long times, the expectation values of the observables in the wave function will be well approximated by the expectation value in this Floquet state.

Second, in this setting, the insights from the single-spin problem studied above are most directly transferable; in particular, we again encounter the ideas of resonances and scars. With these in hand, we can then identify a number of features present in the data more generally, in particular, for high-temperature states (which are of interest in the context of the NMR experiments by Rovny [56]). We find that perturbation theory works best in the vicinity of the scars with their emergent integrability (see below), and we present a limited exploration of the performance of FDPT away from these in Appendix B.

For the expansion of the Floquet state to leading order, the computation proceeds entirely along the lines of that presented for the single-spin model. We denote the state in which all spins $\sigma_{n}^{x}=+1$, except for the site $m$ where $\sigma_{m}^{x}=-1$, as $|m\rangle$. In the limit in which $h_{D}^{x}$ is much larger than $J, \kappa$, and $h_{0}^{x}$, we find that, to leading order in $h^{z} / h_{D}^{x}$, Eq. (B24) takes the form

$$
\begin{aligned}
c_{m}(0) & \simeq \frac{h^{z}}{h_{D}^{x}} \frac{e^{i A T / 2}\left[e^{i h_{D}^{x} T}-\cos (A T / 2)\right]}{e^{i A T}-1}, \\
A & =4(J-\kappa)+2 h_{0}^{x} .
\end{aligned}
$$

The magnetization of this maximally polarized Floquet state is given as follows. The expectation value of $\sum_{n=1}^{L} \sigma_{n}^{x}$ in each of the $m$ states is $L-2$, and in the state $|0\rangle$, it is $L$. This gives

$$
m^{x}=1-\frac{2}{L} \sum_{m=1}^{L}\left|c_{m}(0)\right|^{2}
$$

\section{Resonances and stability of the scar}

The resonance condition [Eqs. (29) and (38)],

$$
e^{i A T}=1 \quad \text { where } A=4(J-\kappa)+2 h_{0}^{x},
$$

signals the singularities of our expansion, where $c_{m}(0)$ diverges. For our Hamiltonian, this occurs for

$$
h_{0}^{x}=-2 J+2 \kappa+\frac{p \omega}{2} .
$$

Here, $p$ is an integer that corresponds to the number of photons absorbed or emitted in this transition. Thus, our first-order theory does not preclude multiphoton transitions.

This suggests considering all possible first-order resonances based on Eq. (29), by considering the resonance condition more generally: Evaluating the change $E_{m}-E_{n}$ due to the flip of only a single spin, $\sigma_{0}$, with $n$th nearestneighbor spins on the right or left denoted by $\sigma_{ \pm n}$, yields the first-order resonance condition

$$
h_{0}^{x} \sigma_{0}+J \sigma_{0}\left(\sigma_{-1}+\sigma_{1}\right)-\kappa \sigma_{0}\left(\sigma_{-2}+\sigma_{2}\right)=\frac{p \omega}{2} .
$$

Of course, individual resonances may be absent if there are no matrix elements between the states in question.

This approach can be rather successful at identifying the locations of the numerically observed isolated resonances, as displayed in Fig. 4. There, the strength of the freezing is displayed as a function of driving strength, for both slow and very slow drives, $\omega=0.4$ and 0.04 , respectively.

The right panel of Fig. 4 emphasizes the generality of this result: The considerations of the first-order resonances obtained above yield the response even for the initially weakly polarized $\left(m^{x}=0.05\right)$, high-temperature initial state.

For a many-body Floquet system, a proliferation of Floquet resonances may lead to unbounded heating. Hence, a stable nonthermal state (e.g., a scar) a priori requires the absence of resonances. Equation (42) shows that this is straightforwardly possible to first order since the resonances are isolated and can be well separated in parameter space. This stems from the fact that the gap $E_{m}-E_{n}$ between two distinct (possibly degenerate) levels of $H_{0}^{x}$ [Eq. (6)] does not necessarily vanish even in the thermodynamic limit (for example, if we take all the couplings in $H_{0}^{x}$ to be rational numbers). The absence of any signature of the higher-order resonances in the exact numerical result at very low frequencies $(\omega=0.04)$ and large $h_{D}^{x}=40$ in the neighborhoods of the scar points indicates that the firstorder theory is sufficient there, and the FDPT series is at least asymptotic in nature.

Higher-order resonances start gaining importance as $h_{D}^{x}$ is reduced below a freezing cutoff $\left(h_{D}^{x} \approx 18\right)$, as shown in Fig. 1(b). The choice of parameters rules out first-order resonances in this case. The results are consistent with this 
(a)

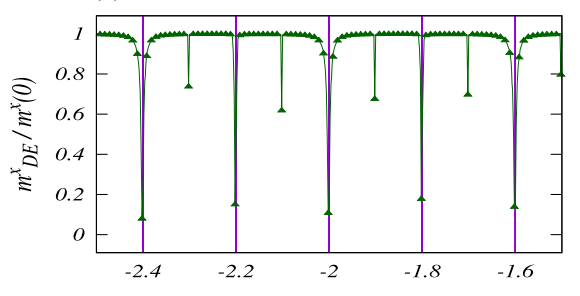

(b)

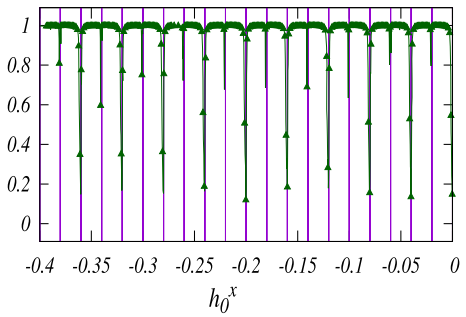

(c)

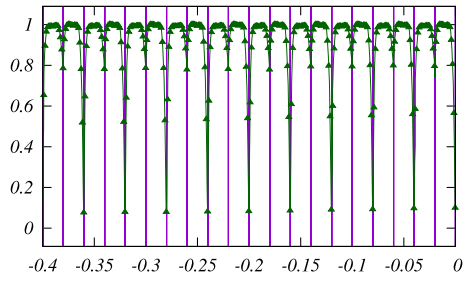

FIG. 4. Interacting case: Freezing and resonances in the magnetization ratio $m_{\mathrm{DE}}^{x} / m_{0}^{x}$ versus $h_{0}^{x}$. The observable, initial states at zero [panels (a,b)] and high temperature (inverse temperature $\beta=10^{-2}$ ) [panel (c)], and other parameters are as described in Fig. 1(a). Results shown for slow [(a) $\omega=0.4]$ and very slow [(b,c) $\omega=0.04]$ drives (points on the green lines). The resonances obtained from first-order FDPT, Eq. (42) (purple vertical lines), show a remarkable match with the numerical values of dips in $m^{x}$. [Some higher-order resonances are also visible at $\omega=0.4$ in panel (a)]. The other parameters are the same as in Fig. 1.

when the drive amplitude is above the cutof-we see no resonant dip in $m_{\mathrm{DE}}^{x}$. But as $h_{D}^{x}$ is tuned below the cutoff, rapid irregular fluctuations appear due to sharp resonant dips in $m_{\mathrm{DE}}^{x}$. With the first-order resonances being ruled out, these dips are due to higher-order resonances, which implies that first-order perturbation theory is insufficient below the cutoff. With further lowering of $h_{x}^{D}$, a sharp drop to the Floquet thermalized regime $m_{\mathrm{DE}}^{x} \sim 0$ eventually appears below a threshold $\left(h_{D}^{x} \approx 5\right)$.

Scar from FDPT.-Considering the expression for the magnetization, obtained by substituting the expression for $c_{m}(0)$ [Eq. (38)] into the expression of $m^{x}$ [Eq. (39)],

$$
\begin{aligned}
1-m^{x}= & 2\left(\frac{h^{z}}{h_{D}^{x}}\right)^{2} \\
& \times \frac{1+\cos ^{2}(A T / 2)-2 \cos (A T / 2) \cos \left(h_{D}^{x} T\right)}{4 \sin ^{2}(A T / 2)},
\end{aligned}
$$

we make the following observations.

First, Eq. (43) indicates that $m^{x}$ should keep oscillating with $h_{D}^{x}$ with a period $\omega$ [except when $\cos (A T / 2)$ is close to zero], as is indeed observed in Fig. 5. Notice, therefore, that the "high-field limit" is not entirely simple but is still endowed with a fine-structured periodicity.

Second, when $\omega=2 \pi / T$ is large, we can approximate $\cos (A T / 2) \simeq 1-(A T)^{2} / 8$ and $\sin (A T / 2) \simeq A T / 2$ in Eq. (43):

$$
1-m^{x}=2\left(\frac{h^{z}}{h_{D}^{x}}\right)^{2} \frac{4\left(1-A^{2} T^{2} / 8\right) \sin ^{2}\left(h_{D}^{x} T / 2\right)}{A^{2} T^{2}} .
$$

This expression shows that freezing becomes weaker with increasing $\omega$. An exception to this occurs when the numerator in Eq. (44) vanishes, namely, when $\omega=h_{D}^{x} / k$, where $k$ is an integer. At these points, we have $m^{x} / m^{x}(0)=1$, i.e., perfect freezing. These points are precisely the "scar" points given by Eq. (7), where the peaks of freezing are obtained numerically (Fig. 1).

As encountered in the single-spin model, there is an interesting interplay between the scars, where $m^{x}$ is frozen, and the resonances, where heating is hugely amplified. When the two coincide, this can destroy the inertness of the scar point, which is manifested as sharp dips in $m_{\mathrm{DE}}^{x}$ in the numerical results discussed above, and for intermediate values of $h_{D}^{x}$ in the inset of Fig. 1(a). The FDPT predicts
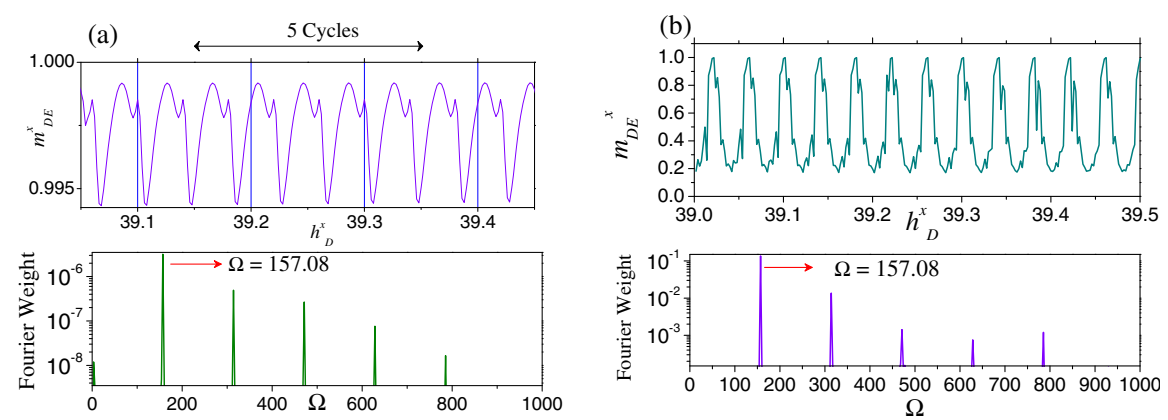

FIG. 5. Periodicity in drive strength, $h_{D}^{x}$, of the magnetization response [diagonal ensemble average $m_{\mathrm{DE}}^{x}$, Eq. (3)]. The top row shows periodicity for both off-resonance (left, $h_{0}^{x}=-0.2$ ) and on-resonance (right, $h_{0}^{x}=-0.21$ ) drives. Other parameters and the initial lowtemperature state, as in Fig. 2(b). In both cases, the leading frequency of oscillations is $\Omega \approx 157.08 \approx 2 \pi / \omega$, visible in the bottom panel, as predicted by Eq. (43). The other parameters are the same as in Fig. 1(a). 
isolated resonances in parameter space and provides a guide for choosing the Hamiltonian parameters to avoid resonances and observe stable scars. Our choice of parameters for Fig. 1 is guided by the theory [Eq. (42)], and we indeed observe resonance-free strong freezing at the scar points.

It would clearly be desirable to embark on a more detailed study, both with respect to the role of higher-order resonances (visible in the left panel of Fig. 4) and with regard to the statistics of the resonances as the system size increases.

\section{ROBUSTNESS AND GENERALITY OF THE SCARRING AND EMERGENT CONSERVATION}

In this section, we demonstrate the robustness and generality of the phenomenon of emergent conservation and the consequent absence of thermalization, by comparing the diagonal ensemble average $m_{\mathrm{DE}}^{x}$ with the driving frequency $\omega$ for a range of qualitatively distinct models. We also demonstrate the stability of the conservation law at the scar or freezing points upon increasing the system size. In all cases, the drive strength is set to be $h_{D}^{x}=40$, and the freezing peaks or scar points are thus expected to occur for $\omega=40 / k$, where $k$ is an integer. This condition was derived for all Ising interactions in Sec. IV and will be derived for general two-body Heisenberg interactions in Sec. VIB.

\section{A. Additional forms of Ising interactions}

First, we confirm, as predicted by the moving-frame Magnus expansion in Sec. IV, the robustness of the phenomenon under diverse variations of the form of $H_{0}^{x}$ in the total Hamiltonian partitioned in the form of Eq. (6). We recall that $H_{0}^{x}$ is the portion of the static part of the Hamiltonian that commutes with $H_{D}$, and the nature of the whole static part can be tuned over a wide variety of many-body Hamiltonians depending on the form of $H_{0}^{x}$, ranging from noninteracting to interacting, integrable to nonintegrable, and low to high dimensional. We consider two forms for $H_{0}^{x}$. First, we add a three-body interaction, of strength $J_{x x x}$,

$$
\begin{aligned}
H_{0}^{x(3 \text { Spin })}= & -J \sum_{i} \sigma_{i}^{x} \sigma_{i+1}^{x}+\kappa \sum_{i} \sigma_{i}^{x} \sigma_{i+2}^{x} \\
& +J_{x x x} \sum_{i} \sigma_{i}^{x} \sigma_{i+1}^{x} \sigma_{i+2}^{x}-h_{0}^{x} \sum_{i}^{L} \sigma_{i}^{x} .
\end{aligned}
$$

Second, we consider long-range interactions as follows. Spins are placed equidistantly on a circle, and the distance $r_{i j}$ between the $i$ th and the $j$ th spin is measured along the chord connecting them, such that

$$
H_{0}^{x(\mathrm{LR})}=-J \sum_{i j} \frac{\sigma_{i}^{x} \sigma_{j}^{x}}{r_{i j}}-h_{0}^{x} \sum_{i}^{L} \sigma_{i}^{x} .
$$

The increased effective coordination number is intended to mimic the phenomenology of higher-dimensional models. The results are given in Fig. 6.

\section{B. General Heisenberg interactions}

We consider the case where the static part of the Hamiltonian $H(t)$ [Eq. (6)] not only consists of a simple transverse field but also includes a general Heisenberg
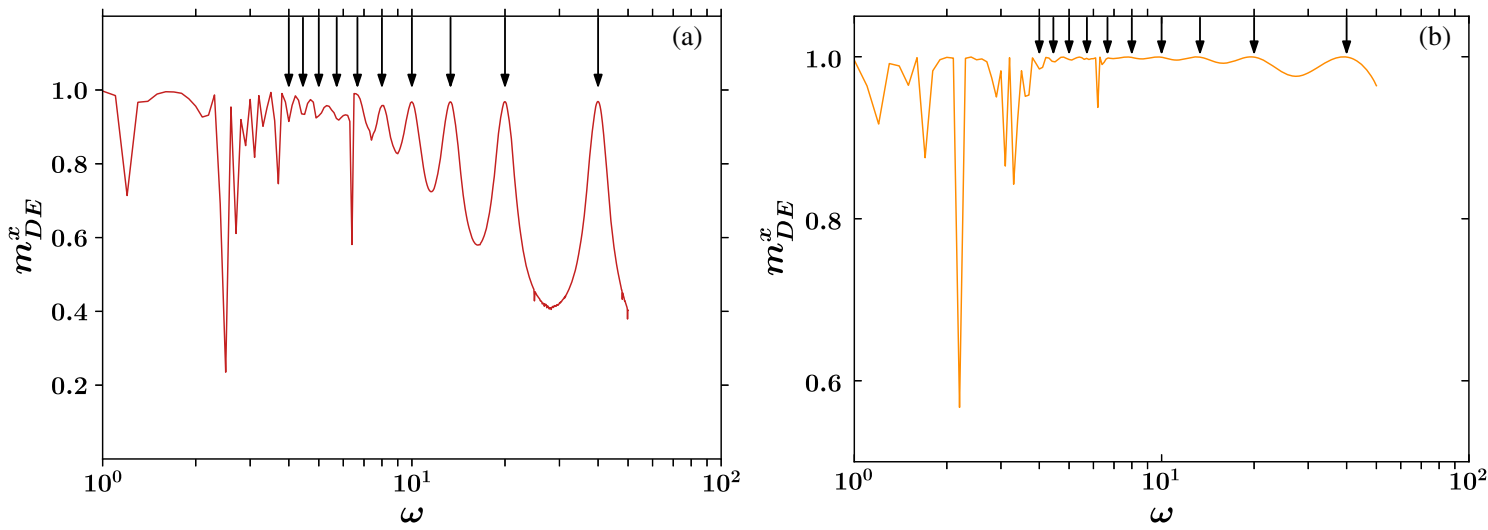

FIG. 6. Infinite-time limit of the magnetization, $m_{\mathrm{DE}}^{x}$, versus driving frequency $\omega$ for Hamiltonians of the form in Eq. (6) but with different types of $H_{0}^{x}$, namely, three-spin interactions [(a) $H_{0}^{x}=H_{0}^{x(3 \mathrm{Spin})}$, Eq. (45)] and long-range interactions [(b) $H_{0}^{x}=H_{0}^{x(\mathrm{LR})}$, Eq. (46)]. The strong freezing of $m_{\mathrm{DE}}^{x}$ near the freezing points $\left(\omega=h_{D}^{x} / k\right)$ is observed in agreement with the prediction of the Magnus expansion up to the two leading orders in both cases. Interestingly, small corrections due to the higher-order terms are also observed, namely, small deviations of the peak heights from unity for the three-spin case, and tiny shifts of the peak from the freezing condition for the long-range case. Apart from these corrections, the higher-order terms do not appear to change any of the key aspects of the phenomenon (strong emergent conservation of $m^{x}$ at all times and consequent lack of unbounded heating). Here, the parameters are $J_{x x x}=0.5, J=1, \kappa=0.7 \pi / 3, h_{0}^{x}=e / 10, h^{z}=1.2, h_{D}^{x}=40, L=20$. 
interaction with arbitrary position dependence. The Heisenberg terms involving $\sigma_{i}^{y, z}$ are included in the $V$ term, and those involving $\sigma_{i}^{x}$ are included in the $H_{x}^{0}$ term as follows. The total Hamiltonian $H(t)=H^{\mathrm{HB}}(t)$, in this case, has the same form in Eq. (6) but with $V$ replaced by

$$
V^{\mathrm{HB}}=-\sum_{i, j} J_{i j}^{y} \sigma_{i}^{y} \sigma_{j}^{y}-\sum_{i, j} J_{i j}^{z} \sigma_{i}^{z} \sigma_{j}^{z}-h^{z} \sum_{i} \sigma^{z}
$$

and $H_{0}^{x}$ replaced by

$$
H_{0}^{x(\mathrm{HB})}=-\sum_{i, j} J_{i j}^{x} \sigma_{i}^{x} \sigma_{j}^{x}+\kappa \sum \sigma_{i}^{x} \sigma_{i+2}^{x}-h_{0}^{x} \sum \sigma_{i}^{x} .
$$

The total static Hamiltonian $V+H_{0}^{x}$ can thus have a general Heisenberg term with an arbitrary interaction graph (coordination number, spatial dimensionality, and position dependence).
For the changed form of $V$, the moving frame Magnus expansion requires some additional lengthy steps (see Appendix A 2) but eventually leads to the same conclusion as derived in Sec. IV; namely, $m^{x}$ is exactly conserved in the first two orders of the expansion.

Interestingly, the first term (zeroth order in $1 / \omega$ ) exhibits an attractive route to the emergent conserved quantity: All the terms, in addition to $H_{0}^{x}$, do not in fact vanish, but their sum explicitly exhibits a U(1) symmetry present neither in $H(t)$ nor in $H^{\mathrm{mov}}(t)$. This assures conservation of $m^{x}$ in the first order. In the next order (first order in $1 / \omega$ ), all the terms except $H_{0}^{x}$ vanish. In the following, we summarize the results, relegating the detailed calculation to Appendix A 1.

For the total Hamiltonian $H^{\mathrm{HB}}(t)$, employing the unitary transformation induced by $W(t)$ [Eq. (13)], we switch to the moving frame, in which our total Hamiltonian reads

$$
\begin{aligned}
H_{\mathrm{HB}}^{\mathrm{mov}}(t)= & H_{0}^{x}-\sum_{i, j} J_{i j}^{y} \sigma_{i}^{y} \sigma_{j}^{y}\left[\mathbb{I} \cos ^{2}(2 \theta)-\sigma_{i}^{x} \sigma_{j}^{x} \sin ^{2}(2 \theta)+\frac{i}{2} \sin (4 \theta)\left(\sigma_{i}^{x}+\sigma_{j}^{x}\right)\right] \\
& -\sum_{i, j} J_{i j}^{z} \sigma_{i}^{z} \sigma_{j}^{z}\left[\mathbb{I} \cos ^{2}(2 \theta)-\sigma_{i}^{x} \sigma_{j}^{x} \sin ^{2}(2 \theta)+\frac{i}{2} \sin (4 \theta)\left(\sigma_{i}^{x}+\sigma_{j}^{x}\right)\right] \\
& -h^{z} \cos (2 \theta) \sum_{i} \sigma_{i}^{z}+h^{z} \sin (2 \theta) \sum_{i} \sigma_{i}^{y} .
\end{aligned}
$$

In the following, we state the results of the Magnus expansion of $H_{\mathrm{HB}}^{\mathrm{mov}}(t)$.

The first term (zeroth order in $1 / \omega$ ) is the average Hamiltonian, given by

$$
\begin{aligned}
H_{\mathrm{eff}}^{(0)} & =\frac{1}{T} \int_{0}^{T} d t H_{\mathrm{HB}}^{\mathrm{mov}}(t) \\
& =H_{0}^{x(\mathrm{HB})}-\frac{1}{2} \sum_{i, j}\left(J_{i j}^{y}+J_{i j}^{z}\right)\left[\sigma_{i}^{y} \sigma_{j}^{y}+\sigma_{i}^{z} \sigma_{j}^{z}\right],
\end{aligned}
$$

under the freezing condition $h_{D}^{x} T=2 \pi k$ ( or $h_{D}^{x}=k \omega$ ). This term, though nontrivial and nonzero, is visibly U(1) symmetric and commutes with $m^{x}$.

The second term (first order in $1 / \omega$ ) reads

$H_{\mathrm{eff}}^{(1)}=\frac{1}{2 !(i) T} \int_{0}^{T} d t_{1} \int_{0}^{t_{1}} d t_{2}\left[H_{\mathrm{HB}}^{\mathrm{mov}}\left(t_{1}\right), H_{\mathrm{HB}}^{\mathrm{mov}}\left(t_{2}\right)\right]$.

Using Eq. (49), calculating all the commutators, and performing the integrals (see Appendix A 2), we finally get, under the freezing condition $h_{D}^{x} T=2 \pi k$,

$$
H_{\text {eff }}^{(1)}=0,
$$

which finally yields

$$
H_{\mathrm{eff}}=H_{0}^{x(\mathrm{HB})}-\frac{1}{2} \sum_{i, j}\left(J_{i j}^{y}+J_{i j}^{z}\right)\left[\sigma_{i}^{y} \sigma_{j}^{y}+\sigma_{i}^{z} \sigma_{j}^{z}\right],
$$

up to $\mathcal{O}\left(1 / \omega^{2}\right)$. This result implies strong conservation of $m^{x}$ for large $h_{D}^{x}$ at the freezing points. We numerically check two well-known special cases, namely, the translationally invariant, isotropic and anisotropic Heisenberg chains (in the presence of all the other interactions considered earlier). The clean, translationally invariant, nonintegrable chains with short-range interactions, as employed here for the demonstration, are probably the easiest to heat up (hence hardest to freeze), enjoying no protection from localization of any sort or additional stability that could occur due to a high coordination number. The results are summarized in Figs. 7(a) and 7(b).

\section{C. $L$ dependence of the freezing}

The $L$ dependence of $m_{\mathrm{DE}}^{x}$ at the scar points throws light on the stability of the emergent conservation with increasing system size. The conservation shows no perceptible degradation with increasing $L$, in agreement with the above analytical result for $\omega=10$. For $\omega=1$, there is a nonmonotonic behavior but no systematic decline. These kinds of irregularities [see, e.g., Figs. 8(c) and 8(d)] often occur as "pathological" finite-size effects close to integrability [57]. 

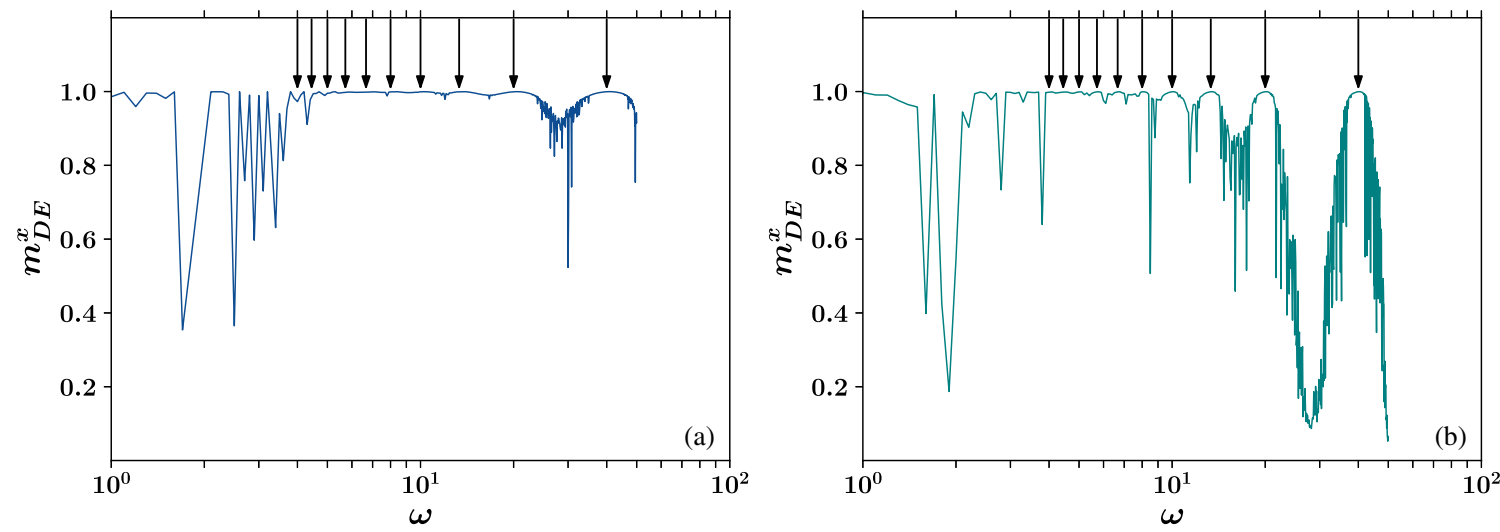

FIG. 7. Infinite-time limit of the magnetization, $m_{\mathrm{DE}}^{x}$, versus driving frequency $\omega$ for Heisenberg interactions. (a) For the Hamiltonian in [Eq. (6)], with $V$ replaced by $V^{\mathrm{HB}}$ [Eq. (47)] and $H_{0}^{x}$ by $H_{0}^{x(\mathrm{HB})}$ [Eq. (48)], with translationally invariant isotropic interaction strengths $\left(J_{i}^{x}=J_{i}^{y}=J_{i}^{z}=1.0\right)$. (b) Same model as in the left panel, but with anisotropic interaction strengths $J_{i}^{x}=1.0, J_{i}^{y}=0.5, J_{i}^{z}=0.6$. In both cases, the emergence conservation of $m^{x}$ and the concomitant absence of thermalization are clearly visible. Slight shifts of the peaks from the predicted values are observed as in Fig. 6. Data are shown for $J=1, \kappa=0.7 \pi / 3, h_{0}^{x}=e / 10, h^{z}=1.2, h_{D}^{x}=40, L=20$.

With present-day numerical methods unable to access significantly larger system sizes, our data cannot, of course, rule out thermalization appearing beyond an asyet-unknown, much larger "prethermal length scale." However, we emphasize that the results in Fig. 8 do not even exhibit a discernible systematic tendency towards unfreezing as a precursor to thermalization as the system size is increased.

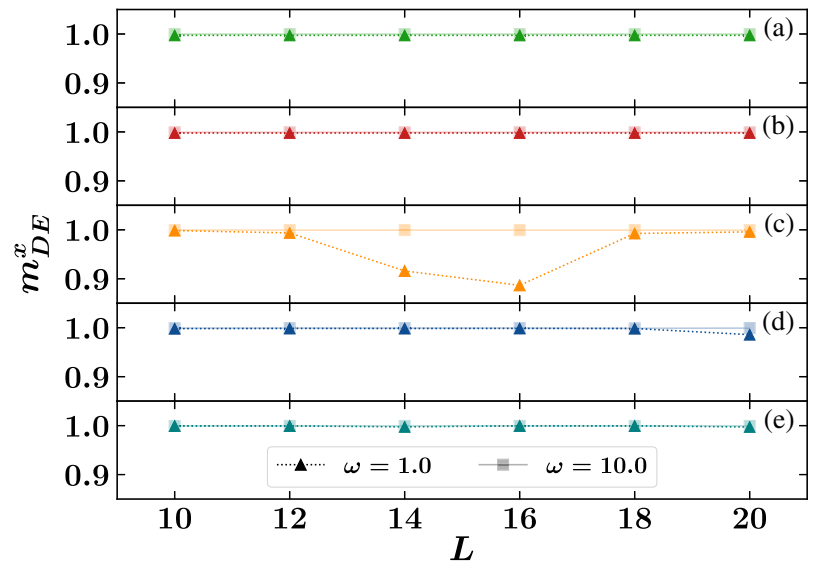

FIG. 8. $\quad L$ dependence of $m_{\mathrm{DE}}^{x}$ at the freezing peaks corresponding to $h_{D}^{x}=40$ and for $\omega=1.0$ and 10.0, plotted against the system size $L$ for different variants of the drive Hamiltonian $H(t)$ [Eq. (6)]. Panels (a)-(c) show different variants of Ising interactions: (a) $H_{0}^{x}$ contains the nearest- and next-nearest-neighbor interactions and on-site fields [Figs. 1(a)]. (b) $H_{0}^{x}=H_{0}^{x(3 \text { Spin })}$ [three-spin interactions; Fig. 6(a)]. (c) $H_{0}^{x}=H_{0}^{x(\mathrm{LR})}$ [additional long-range interactions; Fig. 6(b)]. Panels (d) and (e) show Heisenberg interactions: $H(t)=H^{\mathrm{HB}}(t)$. Results are shown for (d) translationally invariant clean chains and for the isotropic case [Fig. 7(a)] and (e) for the anisotropic case [Fig. 7(b)].

\section{Resonances}

Finally, we note that our treatment of the resonances discussed in Sec. V B 1 will essentially carry over to the expanded settings discussed in this section. In particular, the isolated nature of first-order resonances discussed there, which underpins the stability of the conservation law, remains intact, as follows. For the various forms of $H_{0}^{x}$, Eq. (42) still equates to a finite change in the eigenvalue of $H_{0}^{x}$ due to a single spin flip with an integer multiple of $\omega / 2$. Since the single spin-flip energies remain finite as $L \rightarrow \infty$, the resonances are isolated. For the case of Heisenberg interactions, in our formalism, the Heisenberg terms will be absorbed into the perturbation and not otherwise affect the resonance condition.

These numerical and analytical observations all point towards the emergence of a stable conservation law-not present in the undriven case-and the absence of ergodic heating starting from any generic initial state across a wide range of quantum chaotic systems under a strong periodic drive.

\section{CONCLUSIONS AND OUTLOOK}

In conclusion, we have demonstrated that generic, interacting, Floquet systems subjected to a strong periodic drive can exhibit scar points, i.e., points in the drive parameter space at which the system becomes nonergodic due to the emergence of constraints in the form of a emergent conservation law not present in the undriven system. This manifests itself in the absence of ergodicity and unbounded heating starting from an arbitrary initial state at and around these points, which is captured by our strong-field Magnus expansion in a time-dependent frame. For low drive frequencies, we formulate a novel perturbation theory (Floquet-Dyson perturbation theory) that 
works, even at first order, very accurately at or near integrability of the scar points. In particular, the resonances predicted by the theory accurately coincide with the sharp dips in the emergent conserved quantity. At the resonances, the system absorbs energy without bound from the drive, and hence the scars "compete" with the resonances. The resonances predicted by the theory appear to be isolated in parameter space, and thus, the theory provides a guideline for choosing parameters for observing resonance-free stable scars, as we demonstrate here. These results hold, in particular, for Ising systems in any dimension and with any form of the Ising interactions, as well as in the presence of additional pairwise Heisenberg interactions forming an arbitrary interaction graph. We also demonstrate the robustness of the phenomenon in the presence of anisotropic Heisenberg $(X Y Z)$ interactions.

The exact mechanism of this many-body phenomenon is actually still unknown, and the intuitions we have gathered are based on renormalization of the couplings, which are most effectively revealed under the nonperturbative, time-dependent frame transformation. For certain values of parameters, these renormalization factors vanish owing to destructive many-body quantum interference. These features are not captured by ordinary (lab-frame) Magnus expansion because it misses the effective resummation necessary for these factors to manifest, as performed by the frame transformation.

The emergence and stability of a conservation law in an interacting, quantum, chaotic many-body system due to strong periodic drive is an unexpected and intriguing phenomenon, which warrants extensive investigations. One important direction is to study the nature of the state through continuous (nonstroboscopic) time- - the so-called micromotions. A powerful technique to study this is the so-called van Vleck expansion (also known as the "high-frequency expansion" see, e.g., Refs. $[28,53,58]$ and references therein) of the Floquet Hamiltonian. This method is also a potential alternative to our approach to study the stroboscopic problem.

Our work also touches on various Floquet experiments. In the original experimental work on Floquet many-body localization [31], the interest in a large drive was already noted. In the context of the studies of Floquet time crystals, the two kinds of states studied above have also played a central role: The trapped ion experiment [55] used a fully polarized starting state, while the NMR experiment [56] employed a high-temperature state.

Our work points towards the important role in nonequilibrium settings played by the generation of emergent conservation laws and constraints, in contrast to only focusing on those existing in the static (undriven) system, and their demise under an external drive. Our work also opens a door for stable Floquet engineering in interacting systems and indicates a recipe for tailoring interesting states and structured Hilbert spaces by choosing suitable drive Hamiltonians.

\section{ACKNOWLEDGMENTS}

A. D. thanks Subinay Dasgupta and Sirshendu Bhattacharyya for collaborating on a noninteracting version of the phenomenon studied here [40]. We acknowledge useful discussions with Marin Bukov. The QuSpin PYTHON package $[59,60]$ was used in this work. This research was supported in part by the International Centre for Theoretical Sciences (ICTS) during a visit for the program Thermalization, Many Body Localization and Hydrodynamics (Code: ICTS/hydrodynamics2019/11). A. D. and A. H. acknowledge the partner group program "Spin liquids: Correlations, dynamics and disorder" between IACS and MPI-PKS, and the visitors program of MPI-PKS for supporting visits to PKS during the collaboration. This research was, in part, supported by the Deutsche Forschungsgemeinschaft under the cluster of excellence EXC2147 ct.qmat (Project No. 39085490) and developed with funding from the Defense Advanced Research Projects Agency (DARPA) via the DRINQS program. The views, opinions, and/or findings expressed are those of the authors and should not be interpreted as representing the official views or policies of the Department of Defense or the U.S. Government. R. M. is grateful to Vedika Khemani, David Luitz, and Shivaji Sondhi for collaboration on related work [61]. D. S. thanks DST, India, Project No. SR/S2/JCB-44/2010, for financial support.

\section{APPENDIX A: STRONG-FIELD FLOQUET EXPANSION}

\section{Ising case}

Here, we provide the details of the derivation of the effective Hamiltonian in Eqs. (17)-(20). Carrying out the Pauli algebra gives

$$
\begin{aligned}
H_{\text {mov }} & =H_{0}^{x}-h^{z} \sum_{i}\left[\cos (2 \theta) \sigma_{i}^{z}+\sin (2 \theta) \sigma_{i}^{y}\right], \quad \text { where } \\
\theta(t) & =-h_{D}^{x} \int_{0}^{t} d t^{\prime} \operatorname{Sgn}\left(\sin \omega t^{\prime}\right) .
\end{aligned}
$$

We note that the frame change does not affect $m^{x}$ since it commutes with $W(t)$.

Next, we perform the Magnus expansion of $H_{\mathrm{mov}}$. The initial orders are given by

$$
\begin{aligned}
H_{\mathrm{eff}} & =\sum_{n=0}^{\infty} H_{F}^{0}, \quad \text { where } \\
H_{F}^{(0)} & =\frac{1}{T} \int_{0}^{T} H_{\mathrm{mov}}(t) d t, \\
H_{F}^{(1)} & =\frac{1}{2 ! i T} \int_{0}^{T} d t_{1} \int_{0}^{t_{1}} d t_{2}\left[H_{\mathrm{mov}}\left(t_{1}\right), H_{\mathrm{mov}}\left(t_{2}\right)\right],
\end{aligned}
$$


etc. We first consider the term $H_{F}^{(0)}$. It is easy to check that $H_{0}^{x}$ remains unaffected by the rotation, and the integrals in the first term vanish, giving

$$
H_{F}^{(0)}=H_{0}^{x} .
$$

Next, we consider the second-order term

$$
H_{F}^{(1)}=\frac{1}{2 !(i) T} \int_{0}^{T} d t_{1} \int_{0}^{t_{1}} d t_{2}\left[H\left(t_{1}\right), H\left(t_{2}\right)\right] .
$$

Arranging the terms in the commutator, we get

$$
\begin{aligned}
{\left[H\left(t_{1}\right), H\left(t_{2}\right)\right] } & =K_{1}+K_{2}+K_{3}, \quad \text { where } \\
K_{1} & =-h^{z}\left\{\cos \left(\theta\left(t_{2}\right)\right)-\cos \left(\theta\left(t_{1}\right)\right)\right\}\left[H_{0}^{x}, \mathcal{S}_{z}\right], \\
K_{2} & =-h^{z}\left\{\sin \left(\theta\left(t_{2}\right)\right)-\sin \left(\theta\left(t_{1}\right)\right)\right\}\left[H_{0}^{x}, \mathcal{S}_{y}\right], \\
K_{3} & =\left(h^{z}\right)^{2} \sin \left[\theta\left(t_{2}\right)-\theta\left(t_{1}\right)\right]\left[\mathcal{S}_{z}, \mathcal{S}_{y}\right],
\end{aligned}
$$

where $\mathcal{S}_{x / y / z}=\sum_{i}^{L} \sigma_{i}^{x / y / z}$.

Next, we note that the integral in Eq. (A4) can be broken up in the following way,

$$
\begin{aligned}
I\left[f\left(\theta\left(t_{1}\right), \theta\left(t_{2}\right)\right)\right]= & \int_{0}^{T} d t_{1} \int_{0}^{t_{1}} d t_{2}\left[f\left(\theta\left(t_{1}\right), \theta\left(t_{2}\right)\right)\right] \\
= & I_{1}\left[f\left(\theta\left(t_{1}\right), \theta\left(t_{2}\right)\right)\right]+I_{2}\left[f\left(\theta\left(t_{1}\right), \theta\left(t_{2}\right)\right)\right], \\
& +I_{3}\left[f\left(\theta\left(t_{1}\right), \theta\left(t_{2}\right)\right)\right], \text { where } \\
I_{1}\left[f\left(\theta\left(t_{1}\right), \theta\left(t_{2}\right)\right)\right]= & \int_{0}^{T / 2} d t_{1} \int_{0}^{t_{1}} d t_{2}\left[f\left(\theta\left(t_{1}\right), \theta\left(t_{2}\right)\right)\right], \\
I_{2}\left[f\left(\theta\left(t_{1}\right), \theta\left(t_{2}\right)\right)\right]= & \int_{T / 2}^{T} d t_{1} \int_{0}^{T / 2} d t_{2}\left[f\left(\theta\left(t_{1}\right), \theta\left(t_{2}\right)\right)\right], \\
I_{3}\left[f\left(\theta\left(t_{1}\right), \theta\left(t_{2}\right)\right)\right]= & \int_{T / 2}^{T} d t_{1} \int_{T / 2}^{t_{1}} d t_{2}\left[f\left(\theta\left(t_{1}\right), \theta\left(t_{2}\right)\right)\right] .
\end{aligned}
$$

Finally, we note that
For $I_{1}, \theta\left(t_{1}\right)=-h_{D}^{x} t_{1}, \quad \theta\left(t_{2}\right)=-h_{D}^{x} t_{2}$,

For $I_{2}, \theta\left(t_{1}\right)=-h_{D}^{x}\left(T-t_{1}\right), \quad \theta\left(t_{2}\right)=-h_{D}^{x} t_{2}$,

For $I_{3}, \theta\left(t_{1}\right)=-h_{D}^{x}\left(T-t_{1}\right), \quad \theta\left(t_{2}\right)=-h_{D}^{x}\left(T-t_{2}\right)$.

Using Eqs. (A4)-(A7) and evaluating the integrals, we obtain Eqs. (17)-(20) (see Appendix A 3 for further details).

\section{Heisenberg case}

In the Heisenberg case, the total Hamiltonian $H(t)=$ $H^{\mathrm{HB}}(t)$ has the same form as that in Eq. (6), except with $V$ replaced by

$$
V^{\mathrm{HB}}=-\sum_{i, j} J_{i j}^{y} \sigma_{i}^{y} \sigma_{j}^{y}-\sum_{i, j} J_{i j}^{z} \sigma_{i}^{z} \sigma_{j}^{z}-h^{z} \sum_{i} \sigma^{z}
$$

and $H_{0}^{x}$ replaced by

$H_{0}^{x(\mathrm{HB})}=-J_{i j}^{x} \sum \sigma_{i}^{x} \sigma_{j}^{x}+\kappa \sum \sigma_{i}^{x} \sigma_{i+2}^{x}-h_{0}^{x} \sum \sigma_{i}^{x}$.

Now, following Sec. IV, we switch to the moving frame by acting on the total Hamiltonian $H^{\mathrm{HB}}(t)$, with the unitary transformation given by

$$
\begin{aligned}
V(t) & =\exp \left[i h_{D}^{x} \sum_{j} \sigma_{j}^{x} \int_{t_{0}}^{t} \operatorname{Sgn}\left(\sin \left(\omega t^{\prime}\right)\right) d t^{\prime}\right] \\
& =\prod_{j} \exp \left[i h_{D}^{x} \sigma_{j}^{x} \int_{t_{0}}^{t} \operatorname{Sgn}\left(\sin \left(\omega t^{\prime}\right)\right) d t^{\prime}\right],
\end{aligned}
$$

where

$$
\theta(t)=h_{D}^{x} \int_{t_{0}}^{t} \operatorname{Sgn}\left(\sin \left(\omega t^{\prime}\right)\right) d t^{\prime} .
$$

This transformation gives our moving-frame Hamiltonian

$$
\begin{aligned}
H_{\mathrm{HB}}^{\mathrm{mov}}(t)= & \prod_{i} \exp \left[-i \sigma_{i}^{x} \theta(t)\right] H_{0} \exp \left[i \sigma_{i}^{x} \theta(t)\right] \\
= & H_{0}^{x(\mathrm{HB})}-\prod_{i} \exp \left[-i \sigma_{i}^{x} \theta(t)\right]\left[\sum_{k, l} J_{k, l}^{y} \sigma_{k}^{y} \sigma_{l}^{y}\right] \prod_{j} \exp \left[i \sigma_{j}^{x} \theta(t)\right]-\prod_{i} \exp \left[-i \sigma_{i}^{x} \theta(t)\right]\left[\sum_{k, l} J_{k, l}^{z} \sigma_{k}^{z} \sigma_{l}^{z}\right] \prod_{j} \exp \left[i \sigma_{j}^{x} \theta(t)\right] \\
& -h_{z} \prod_{i} \exp \left[-i \sigma_{i}^{x} \theta(t)\right]\left[\sum_{k, l} \sigma_{k}^{z}\right] \prod_{j} \exp \left[i \sigma_{j}^{x} \theta(t)\right]
\end{aligned}
$$

where

$$
H_{0}^{x(\mathrm{HB})}=-J_{i j}^{x} \sum \sigma_{i}^{x} \sigma_{j}^{x}+\kappa \sum \sigma_{i}^{x} \sigma_{i+2}^{x}-h_{0}^{x} \sum \sigma_{i}^{x},
$$

which gives 


$$
\begin{aligned}
H_{\mathrm{HB}}^{\mathrm{mov}}(t)= & H_{0}^{x(\mathrm{HB})}-\sum_{k, l} J_{k, l}^{y} e^{-i \sigma_{k}^{x} \theta(t)} e^{-i \sigma_{l}^{x} \theta(t)}\left(\sigma_{k}^{y} \sigma_{l}^{y}\right) e^{i \sigma_{k}^{x} \theta(t)} e^{i \sigma_{l}^{x} \theta(t)} \\
& -\sum_{k, l} J_{k, l}^{z} e^{-i \sigma_{k}^{x} \theta(t)} e^{-i \sigma_{l}^{x} \theta(t)}\left(\sigma_{k}^{z} \sigma_{l}^{z}\right) e^{i \sigma_{k}^{x} \theta(t)} e^{i \sigma_{l}^{x} \theta(t)}-h^{z} \sum_{k, l} e^{-i \sigma_{k}^{x} \theta(t)} \sigma_{k}^{z} e^{i \sigma_{k}^{x} \theta(t)}
\end{aligned}
$$

The $J_{i j}^{y}$ term can be simplified to

$$
\begin{aligned}
& =-\sum_{i, j} J_{i j}^{y} \sigma_{i}^{y} \sigma_{j}^{y} e^{2 i \sigma_{i}^{x} \theta(t)} e^{2 i \sigma_{j}^{x} \theta(t)} \\
& =-\sum_{i, j} J_{i j}^{y} \sigma_{i}^{y} \sigma_{j}^{y}\left[\mathbb{I} \cos ^{2}(2 \theta)-\sigma_{i}^{x} \sigma_{j}^{x} \sin ^{2}(2 \theta)+i \sin (2 \theta) \cos (2 \theta)\left(\sigma_{i}^{x}+\sigma_{j}^{x}\right)\right] .
\end{aligned}
$$

Similarly, the $J_{i j}^{z}$ term becomes

$$
=-\sum_{i, j} J_{i j}^{z} \sigma_{i}^{z} \sigma_{j}^{z}\left[\mathbb{I} \cos ^{2}(2 \theta)-\sigma_{i}^{x} \sigma_{j}^{x} \sin ^{2}(2 \theta)+i \sin (2 \theta) \cos (2 \theta)\left(\sigma_{i}^{x}+\sigma_{j}^{x}\right)\right] .
$$

The $h_{z}$ term is similar to our previous case, namely,

$$
=-h^{z} \cos (2 \theta) \sum_{i} \sigma_{i}^{z}+h^{z} \sin (2 \theta) \sum_{i} \sigma_{i}^{y} .
$$

Hence,

$$
\begin{aligned}
H_{\mathrm{HB}}^{\mathrm{mov}}(t)= & H_{0}^{x(\mathrm{HB})}-\sum_{i, j} J_{i j}^{y} \sigma_{i}^{y} \sigma_{j}^{y}\left[\mathbb{I} \cos ^{2}(2 \theta)-\sigma_{i}^{x} \sigma_{j}^{x} \sin ^{2}(2 \theta)+\frac{i}{2} \sin (4 \theta)\left(\sigma_{i}^{x}+\sigma_{j}^{x}\right)\right] \\
& -\sum_{i, j} J_{i j}^{z} \sigma_{i}^{z} \sigma_{j}^{z}\left[\mathbb{I} \cos ^{2}(2 \theta)-\sigma_{i}^{x} \sigma_{j}^{x} \sin ^{2}(2 \theta)+\frac{i}{2} \sin (4 \theta)\left(\sigma_{i}^{x}+\sigma_{j}^{x}\right)\right] \\
& -h^{z} \cos (2 \theta) \sum_{i} \sigma_{i}^{z}+h^{z} \sin (2 \theta) \sum_{i} \sigma_{i}^{y} .
\end{aligned}
$$

Next, we perform the Magnus expansion on Eq. (A18). The zeroth-order term is

$$
H_{\mathrm{eff}}^{(0)}=\frac{1}{T} \int_{0}^{T} d t H_{\mathrm{HB}}^{\mathrm{mov}}(t) .
$$

Now, with the definition of $\theta(t)$ given in Eq. (A11), we get

$$
\begin{aligned}
\int_{0}^{T} \cos ^{2}(2 \theta) d t & =\frac{T}{2}+\frac{\sin 2 h_{D}^{x} T}{4 h_{x}^{D}}, \\
\int_{0}^{T} \sin ^{2}(2 \theta) d t & =\frac{T}{2}-\frac{\sin 2 h_{D}^{x} T}{4 h_{x}^{D}}, \\
\int_{0}^{T} \sin (4 \theta) d t & =\frac{1}{4 h_{x}^{D}}\left(1-\cos 2 h_{D}^{x} T\right), \\
\int_{0}^{T} \sin (2 \theta) d t & =\frac{1}{h_{x}^{D}}\left(1-\cos h_{D}^{x} T\right), \\
\int_{0}^{T} \cos (2 \theta) d t & =\frac{1}{h_{D}^{x}} \sin \left(h_{D}^{x} T\right) .
\end{aligned}
$$

Applying the freezing condition,

$$
h_{D}^{x} T=2 \pi n,
$$

we get

$$
\begin{gathered}
\int_{0}^{T} \cos ^{2}(2 \theta) d t=\frac{T}{2}, \\
\int_{0}^{T} \sin ^{2}(2 \theta) d t=\frac{T}{2}, \\
\int_{0}^{T} \sin (4 \theta) d t=0, \\
\int_{0}^{T} \sin (2 \theta) d t=0, \\
\int_{0}^{T} \cos (2 \theta) d t=0 .
\end{gathered}
$$

Putting everything in Eq. (A19), we obtain 


$$
\begin{aligned}
H_{\mathrm{eff}}^{(0)}= & H_{0}^{x(\mathrm{HB})}-\sum_{i, j} J_{i j}^{y} \sigma_{i}^{y} \sigma_{j}^{y} \frac{1}{T}\left[\frac{T}{2}-\sigma_{i}^{x} \sigma_{j}^{x} \frac{T}{2}\right] \\
& -\sum_{i, j} J_{i j}^{z} \sigma_{i}^{z} \sigma_{j}^{z} \frac{1}{T}\left[\frac{T}{2}-\sigma_{i}^{x} \sigma_{j}^{x} \frac{T}{2}\right] \\
= & H_{0}^{x(\mathrm{HB})}-\frac{1}{2} \sum_{i, j}\left[\sigma_{i}^{y} \sigma_{j}^{y}+\sigma_{i}^{z} \sigma_{j}^{z}\right]\left(J_{i j}^{y}+J_{i j}^{z}\right) .
\end{aligned}
$$

We already have

$$
\left[H_{0}^{x(\mathrm{HB})}, m^{x}\right]=0
$$

and one can easily show that

$$
\left[\sum_{i, j}\left(\sigma_{i}^{y} \sigma_{j}^{y}+\sigma_{i}^{z} \sigma_{j}^{z}\right), m^{x}\right]=0 .
$$

The first-order term of the Magnus expansion is

$$
H_{\mathrm{eff}}^{(1)}=\frac{1}{2 ! T i} \int_{0}^{T} d t_{1} \int_{0}^{t_{1}} d t_{2}\left[H_{\mathrm{HB}}^{\mathrm{mov}}\left(t_{1}\right), H_{\mathrm{HB}}^{\mathrm{mov}}\left(t_{2}\right)\right] .
$$

Rearranging all the terms in Eq. (A18), we can write

$$
\begin{aligned}
H_{\mathrm{HB}}^{\mathrm{mov}}(t)= & H_{0}^{x(\mathrm{HB})}+A \cos ^{2}(2 \theta)+B \sin ^{2}(2 \theta) \\
& +C \sin (4 \theta)+D \cos (2 \theta)+E \sin (2 \theta) .
\end{aligned}
$$

Hence,

$$
\begin{aligned}
{\left[H_{\mathrm{HB}}^{\mathrm{mov}}\left(t_{1}\right), H_{\mathrm{HB}}^{\mathrm{mov}}\left(t_{2}\right)\right]=} & {\left[H_{0}^{x(\mathrm{HB})}, A\right] I_{1}+\left[H_{0}^{x(\mathrm{HB})}, B\right] I_{2}+\left[H_{0}^{x(\mathrm{HB})}, C\right] I_{3}+\left[H_{0}^{x(\mathrm{HB})}, D\right] I_{4}+\left[H_{0}^{x(\mathrm{HB})}, E\right] I_{5}+[A, B] I_{6}+[A, C] I_{7} } \\
& +[A, D] I_{8}+[A, E] I_{9}+[B, C] I_{10}+[B, D] I_{11}+[B, E] I_{12}+[C, D] I_{13}+[C, E] I_{14}+[D, E] I_{15},
\end{aligned}
$$

where

$$
\begin{aligned}
& A=-\sum_{i, j}\left[J_{i j}^{y} \sigma_{i}^{y} \sigma_{j}^{y}+J_{i j}^{z} \sigma_{i}^{z} \sigma_{j}^{z}\right], \quad B=\sum_{i, j}\left[\left(J_{i j}^{y} \sigma_{i}^{y} \sigma_{j}^{y}+J_{i j}^{z} \sigma_{i}^{z} \sigma_{j}^{z}\right) \sigma_{i}^{x} \sigma_{j}^{x}\right], \quad C=-\sum_{i, j} \frac{i}{2}\left[\left(J_{i j}^{y} \sigma_{i}^{y} \sigma_{j}^{y}+J_{i j}^{z} \sigma_{i}^{z} \sigma_{j}^{z}\right)\left(\sigma_{i}^{x}+\sigma_{j}^{x}\right)\right], \\
& D=-h^{z} \sum_{i} \sigma_{i}^{z}, \quad \text { and } \quad E=h^{z} \sum_{i} \sigma_{i}^{y},
\end{aligned}
$$

and

$$
\begin{aligned}
I_{1} & =\cos ^{2}\left(2 \theta\left(t_{2}\right)\right)-\cos ^{2}\left(2 \theta\left(t_{1}\right)\right), \\
I_{2} & =\sin ^{2}\left(2 \theta\left(t_{2}\right)\right)-\sin ^{2}\left(2 \theta\left(t_{1}\right)\right), \\
I_{3} & =\sin \left(4 \theta\left(t_{2}\right)\right)-\sin \left(4 \theta\left(t_{1}\right)\right), \\
I_{4} & =\cos \left(2 \theta\left(t_{2}\right)\right)-\cos \left(2 \theta\left(t_{1}\right)\right), \\
I_{5} & =\sin \left(2 \theta\left(t_{2}\right)\right)-\sin \left(2 \theta\left(t_{1}\right)\right), \\
I_{6} & =\cos ^{2}\left(2 \theta\left(t_{1}\right)\right) \sin ^{2}\left(2 \theta\left(t_{2}\right)\right)-\sin ^{2}\left(2 \theta\left(t_{1}\right)\right) \cos ^{2}\left(2 \theta\left(t_{2}\right)\right), \\
I_{7} & =\cos ^{2}\left(2 \theta\left(t_{1}\right)\right) \sin \left(4 \theta\left(t_{2}\right)\right)-\sin \left(4 \theta\left(t_{1}\right)\right) \cos ^{2}\left(2 \theta\left(t_{2}\right)\right), \\
I_{8} & =\cos ^{2}\left(2 \theta\left(t_{1}\right)\right) \cos \left(2 \theta\left(t_{2}\right)\right)-\cos \left(2 \theta\left(t_{1}\right)\right) \cos ^{2}\left(2 \theta\left(t_{2}\right)\right), \\
I_{9} & =\cos ^{2}\left(2 \theta\left(t_{1}\right)\right) \sin \left(2 \theta\left(t_{2}\right)\right)-\sin \left(2 \theta\left(t_{1}\right)\right) \cos ^{2}\left(2 \theta\left(t_{2}\right)\right), \\
I_{10} & =\sin ^{2}\left(2 \theta\left(t_{1}\right)\right) \sin \left(4 \theta\left(t_{2}\right)\right)-\sin \left(4 \theta\left(t_{1}\right)\right) \sin ^{2}\left(2 \theta\left(t_{2}\right)\right), \\
I_{11} & =\sin ^{2}\left(2 \theta\left(t_{1}\right)\right) \cos \left(2 \theta\left(t_{2}\right)\right)-\cos \left(2 \theta\left(t_{1}\right)\right) \sin ^{2}\left(2 \theta\left(t_{2}\right)\right), \\
I_{12} & =\sin { }^{2}\left(2 \theta\left(t_{1}\right)\right) \sin \left(2 \theta\left(t_{2}\right)\right)-\sin \left(2 \theta\left(t_{1}\right)\right) \sin ^{2}\left(2 \theta\left(t_{2}\right)\right), \\
I_{13} & =\sin \left(4 \theta\left(t_{1}\right)\right) \cos \left(2 \theta\left(t_{2}\right)\right)-\cos \left(2 \theta\left(t_{1}\right)\right) \sin ^{2} \theta\left(t_{2}\right), \\
I_{14} & =\sin \left(4 \theta\left(t_{1}\right)\right) \sin \left(2 \theta\left(t_{2}\right)\right)-\sin \left(2 \theta\left(t_{1}\right)\right) \sin \left(4 \theta\left(t_{2}\right)\right), \\
I_{15} & =\sin \left[2\left(\theta\left(t_{1}\right)-\theta\left(t_{2}\right)\right)\right] .
\end{aligned}
$$

Now, one can show that all 15 integrals of $I_{n}$ in Eq. (A30) vanish for the freezing condition. Hence,

$$
H_{\mathrm{eff}}=H_{0}^{x(\mathrm{HB})}-\frac{1}{2} \sum_{i, j}\left[\sigma_{i}^{y} \sigma_{j}^{y}+\sigma_{i}^{z} \sigma_{j}^{z}\right]\left(J_{i j}^{y}+J_{i j}^{z}\right)
$$

up to the first two orders.

\section{Explicit calculation of the integrals in the moving frame Magnus expansion: Ising case (self-contained)}

The Hamiltonian can be written as

$$
H(t)=H_{0}+r(t) H_{D}
$$

We move to the rotating frame using the transformation

$$
H^{\mathrm{mov}}(t)=W^{\dagger}(t) H_{0} W(t),
$$

where the rotation operator is

$$
W(t)=\exp \left[-i \int_{t_{0}}^{t} r\left(t^{\prime}\right) d t^{\prime} H_{D}\right] .
$$


The first case is an Ising model with next-nearest-neighbor terms:

$$
\begin{aligned}
& H_{0}=-\sum_{i} J \sigma_{i}^{x} \sigma_{i+1}^{x}+\kappa \sum \sigma_{i}^{x} \sigma_{i+2}^{x}-h_{0}^{x} \sum \sigma_{i}^{x}-h^{z} \sum \sigma_{i}^{z}, \\
& =H_{0}^{x}+V \\
& H_{D}=-h_{D}^{x} \sum \sigma_{i}^{x},
\end{aligned}
$$

and

$$
r(t)=\operatorname{Sgn}(\sin (\omega t)) .
$$

From Eqs. (A33), (A36), and (A37), we get

$$
\begin{aligned}
W(t) & =\exp \left[i h_{D}^{x} \sum_{j} \sigma_{j}^{x} \int_{t_{0}}^{t} \operatorname{Sgn}\left(\sin \left(\omega t^{\prime}\right)\right) d t^{\prime}\right] \\
& =\prod_{j} \exp \left[i h_{D}^{x} \sigma_{j}^{x} \int_{t_{0}}^{t} \operatorname{Sgn}\left(\sin \left(\omega t^{\prime}\right)\right) d t^{\prime}\right] .
\end{aligned}
$$

Defining

$$
\theta(t)=-h_{D}^{x} \int_{t_{0}}^{t} \operatorname{Sgn}\left(\sin \left(\omega t^{\prime}\right)\right) d t^{\prime}
$$

and putting all of these together, we get

$$
\begin{aligned}
H^{\mathrm{mov}}(t)= & \prod_{i} \exp \left[-i \sigma_{i}^{x} \theta(t)\right] H_{0} \exp \left[i \sigma_{i}^{x} \theta(t)\right] \\
= & H_{0}^{x}-h_{z} \prod_{i} \exp \left[-i \sigma_{i}^{x} \theta(t)\right]\left[\sum_{k} \sigma_{k}^{z}\right] \\
& \times \prod_{j} \exp \left[i \sigma_{j}^{x} \theta(t)\right] \\
= & H_{0}^{x}-h^{z} \sum_{k} e^{-i \sigma_{k}^{x} \theta(t)} \sigma_{k}^{z} e^{i \sigma_{k}^{x} \theta(t)}
\end{aligned}
$$

$\therefore H^{\mathrm{mov}}(t)=H_{0}^{x}-h^{z} \cos 2 \theta \sum_{i} \sigma_{i}^{z}-h^{z} \sin 2 \theta \sum_{i} \sigma_{i}^{y}$.

Now, we can perform Magnus expansion on Eq. (A42). The zeroth-order term is

$$
\begin{aligned}
H_{\mathrm{eff}}^{(0)}= & \frac{1}{T} \int_{0}^{T} H^{\mathrm{mov}}(t) d t \\
= & \frac{1}{T} \int_{0}^{T} H_{0}^{x} d t-\frac{h^{z}}{T} \sum_{i} \sigma_{i}^{z} \int_{0}^{T} \cos 2 \theta d t \\
& -\frac{h^{z}}{T} \sum_{i} \sigma_{i}^{y} \int_{0}^{T} \sin 2 \theta d t .
\end{aligned}
$$

Then, with the definition of $\theta(t)$ as given in Eq. (A39) [note: $\theta(t)=-h_{D}^{x} t$ for $0<t \leq(T / 2)$, and $\theta(t)=$ $-\left(h_{D}^{x} T-h_{D}^{x} t\right) \quad$ for $\left.\quad(T / 2) \leq t \leq T\right]$, the integral simplifies to

$$
\begin{aligned}
\int_{0}^{T} \cos 2 \theta d t & =\int_{0}^{\frac{T}{2}} \cos 2 \theta d t+\int_{\frac{T}{2}}^{T} \cos 2 \theta d t \\
& =\int_{0}^{\frac{T}{2}} \cos \left(2 h_{D}^{x} t\right) d t+\int_{\frac{T}{2}}^{T} \cos 2\left(h_{D}^{x} T-h_{D}^{x} t\right) d t \\
& =\frac{1}{h_{D}^{x}} \sin \left(h_{D}^{x} T\right) .
\end{aligned}
$$

Similarly,

$$
\int_{0}^{T} \sin 2 \theta d t=\frac{1}{h_{D}^{x}}\left(\cos \left(h_{D}^{x} T\right)-1\right) .
$$

Putting Eqs. (A44) and (A45) into Eq. (A43), we get

$$
\begin{aligned}
H_{\mathrm{eff}}^{(0)}= & H_{0}^{x}-\frac{h^{z}}{h_{D}^{x} T} \sum_{i} \sigma_{i}^{z} \sin \left(h_{D}^{x} T\right) \\
& +\frac{h^{z}}{h_{D}^{x} T} \sum_{i} \sigma_{i}^{y}\left(1-\cos \left(h_{D}^{x} T\right)\right) .
\end{aligned}
$$

Now, putting the freezing condition $h_{D}^{x} T=2 n \pi$ in Eq. (A46), i.e., $\sin h_{D}^{x} T=0$ and $\cos h_{D}^{x} T=1$, one gets

$$
\left.H_{\mathrm{eff}}^{(0)}\right|_{\text {freezing }}=H_{0}^{x} .
$$

Next, we evaluate the first-order term,

$$
H_{\text {eff }}^{(1)}=\frac{1}{2 ! T i} \int_{0}^{T} d t_{1} \int_{0}^{t_{1}} d t_{2}\left[H^{\mathrm{mov}}\left(t_{1}\right), H^{\mathrm{mov}}\left(t_{2}\right)\right]
$$

Calling $\theta\left(t_{1}\right)=\theta_{1} ; \quad \theta\left(t_{2}\right)=\theta_{2} \quad$ and $\quad \sum_{i} \sigma_{i}^{z}=S^{z} ;$ $\sum_{i} \sigma_{i}^{y}=S^{y}$ and using the form of $H^{\mathrm{mov}}$ from Eq. (A42), the commutator in Eq. (A48) simplifies to

$$
\begin{aligned}
{\left[H^{\mathrm{mov}}\left(t_{1}\right), H^{\mathrm{mov}}\left(t_{2}\right)\right]=} & {\left[S^{z}, H_{0}^{x}\right] h^{z}\left(\cos 2 \theta_{2}-\cos 2 \theta_{1}\right) } \\
& +\left[S^{y}, H_{0}^{x}\right] h^{z}\left(\sin 2 \theta_{2}-\sin 2 \theta_{1}\right) \\
& +\left[S^{y}, S^{z}\right]\left(h^{z}\right)^{2} \sin \left(2 \theta_{1}-2 \theta_{2}\right) .
\end{aligned}
$$


Now, for example, the integral corresponding to the first term is

$$
\begin{aligned}
& I_{1}= \int_{0}^{T} \int_{0}^{t_{1}} d t_{1} d t_{2}\left(\cos 2 \theta_{2}-\cos 2 \theta_{1}\right) \\
&= \int_{0}^{\frac{T}{2}} d t_{1} \int_{0}^{t_{1}} d t_{2} \cos 2 \theta\left(t_{2}\right)+\int_{\frac{T}{2}}^{T} d t_{1} \int_{0}^{\frac{T}{2}} d t_{2} \cos 2 \theta\left(t_{2}\right)+\int_{\frac{T}{2}}^{T} d t_{1} \int_{\frac{T}{2}}^{t_{1}} d t_{2} \cos 2 \theta\left(t_{2}\right)-\int_{0}^{T} d t_{1} \cos 2 \theta\left(t_{1}\right) t_{1} \\
&= \int_{0}^{\frac{T}{2}} d t_{1} \int_{0}^{t_{1}} d t_{2} \cos \left(2 h_{D}^{x} t_{2}\right)+\int_{\frac{T}{2}}^{T} d t_{1} \int_{0}^{\frac{T}{2}} d t_{2} \cos \left(2 h_{D}^{x} t_{2}\right)+\int_{\frac{T}{2}}^{T} d t_{1} \int_{\frac{T}{2}}^{t_{1}} d t_{2} \cos \left(2 h_{D}^{x} T-2 h_{D}^{x} t_{2}\right) \\
&-\int_{0}^{\frac{T}{2}} d t_{1} t_{1} \cos \left(2 h_{D}^{x} t_{1}\right)-\int_{\frac{T}{2}}^{T} d t_{1} t_{1} \cos \left(2 h_{D}^{x} T-2 h_{D}^{x} t_{1}\right), \\
& I_{1}=I_{1}^{A}+I_{1}^{B}+I_{1}^{C}-I_{1}^{D}-I_{1}^{E}
\end{aligned}
$$

Then,

$$
\begin{gathered}
I_{1}^{A}=\int_{0}^{\frac{T}{2}} d t_{1} \int_{0}^{t_{1}} d t_{2} \cos \left(2 h_{D}^{x} t_{2}\right)=\frac{1}{2 h_{D}^{x}} \int_{0}^{\frac{T}{2}} d t_{1} \sin 2 h_{D}^{x} t_{1}=\frac{1}{\left(2 h_{D}^{x}\right)^{2}}\left[1-\cos h_{D}^{x} T\right] \\
I_{1}^{B}=\int_{\frac{T}{2}}^{T} d t_{1} \int_{0}^{\frac{T}{2}} d t_{2} \cos \left(2 h_{D}^{x} t_{2}\right)=\frac{1}{2 h_{D}^{x}} \int_{\frac{T}{2}}^{T} d t_{1} \sin h_{D}^{x} T=\frac{T}{4 h_{D}^{x}} \sin h_{D}^{x} T \\
I_{1}^{C}=\int_{\frac{T}{2}}^{T} d t_{1} \int_{\frac{T}{2}}^{t_{1}} d t_{2} \cos \left(2 h_{D}^{x} T-2 h_{D}^{x} t_{2}\right)=\frac{1}{2 h_{D}^{x}} \int_{\frac{T}{2}}^{T} d t_{1}\left[\sin h_{D}^{x} T-\sin \left(2 h_{D}^{x} T-2 h_{D}^{x} t_{1}\right)\right] \\
=\frac{T}{4 h_{D}^{x}} \sin h_{D}^{x} T-\frac{1}{\left(2 h_{D}^{x}\right)^{2}}+\frac{1}{\left(2 h_{D}^{x}\right)^{2}} \cos h_{D}^{x} T \\
I_{1}^{D}=\int_{0}^{\frac{T}{2}} d t_{1} t_{1} \cos \left(2 h_{D}^{x} t_{1}\right)=\frac{1}{2 h_{D}^{x}}\left(\frac{T}{2} \sin h_{D}^{x} T-\int_{0}^{\frac{T}{2}} d t_{1} \sin 2 h_{D}^{x} t_{1}\right) \\
I_{1}^{E}=\frac{T}{4 h_{D}^{x}} \sin h_{D}^{x} T+\frac{1}{\left(2 h_{D}^{x}\right)^{2}} \cos h_{D}^{x} T-\frac{1}{\left(2 h_{D}^{x}\right)^{2}}, \\
=\frac{T}{4 h_{D}^{x}} \sin h_{D}^{x} T+\frac{1}{\left(2 h_{D}^{x}\right)^{2}}\left(1-\cos h_{D}^{x} T\right)
\end{gathered}
$$

Now, putting Eqs. (A53)-(A57) into Eq. (A52) yields

$$
I_{1}=0
$$

Carrying out the integrals corresponding to the other two commutators in Eq. (A49), one can similarly get

$$
\begin{gathered}
I_{2}=\int_{0}^{T} \int_{0}^{t_{1}} d t_{1} d t_{2}\left(\sin 2 \theta_{1}-\sin 2 \theta_{2}\right)=0, \\
I_{3}=\int_{0}^{T} \int_{0}^{t_{1}} d t_{1} d t_{2} \sin \left(2 \theta_{1}-2 \theta_{2}\right)=0 .
\end{gathered}
$$

\section{APPENDIX B: FLOQUET-DYSON PERTURBATION THEORY}

We start from Eq. (24), which implies that

$$
\begin{aligned}
& i \sum_{m} \dot{c}_{m}(t) e^{-i \int_{0}^{t} d t^{\prime} E_{m}\left(t^{\prime}\right)}|m\rangle \\
& \quad=V \sum_{m} c_{m}(t) e^{-i \int_{0}^{t} d t^{\prime} E_{m}\left(t^{\prime}\right)}|m\rangle,
\end{aligned}
$$

where the dot over $c_{m}$ denotes $d / d t$. Taking the inner product of Eq. (B1) with $\langle n|$ and using Eq. (23), we find, to first order in $V$, that 


$$
\dot{c}_{n}=0
$$

We can therefore choose

$$
c_{n}(t)=1
$$

for all $t$. We thus have

$$
\left|\psi_{n}(t)\right\rangle=e^{-i \int_{0}^{t} d t^{\prime} E_{n}\left(t^{\prime}\right)}|n\rangle+\sum_{m \neq n} c_{m}(t) e^{-i \int_{0}^{t} d t^{\prime} E_{m}\left(t^{\prime}\right)}|m\rangle .
$$

Hence, Eq. (25) implies that the Floquet eigenvalue is still given by $\mu_{n}^{(0)}=\int_{0}^{T} d t E_{n}(t)$ up to first order in $V$.

Next, taking the inner product of Eq. (B1) with $\langle m|$, where $m \neq n$, we find, to first order in $V$, that

$$
\dot{c}_{m}=-i\langle m|V| n\rangle e^{i \int_{0}^{t} d t^{\prime}\left[E_{m}\left(t^{\prime}\right)-E_{n}\left(t^{\prime}\right)\right]},
$$

so

$$
c_{m}(T)=c_{m}(0)-i\langle m|V| n\rangle \times \int_{0}^{T} d t e^{i \int_{0}^{t} d t^{\prime}\left[E_{m}\left(t^{\prime}\right)-E_{n}\left(t^{\prime}\right)\right]} .
$$

We now impose the condition on $\left|\psi_{n}(T)\right\rangle$ of Eq. (26) such that $\left|\psi_{n}(0)\right\rangle$ turns out to be a Floquet state; i.e., from Eq. (B4), we must have

$$
\psi_{n}(T)=e^{-i \int_{0}^{T} d t E_{n}(t)} \psi_{n}(0),
$$

namely, we must have

$$
c_{m}(T)=e^{i \int_{0}^{T} d t\left[E_{m}(t)-E_{n}(t)\right]} c_{m}(0)
$$

for all $m \neq n$. Clearly, $\left|\psi_{n}(0)\right\rangle$ satisfying this condition can be identified as the Floquet state $\left|\mu_{n}\right\rangle$.

\section{Single-spin model}

\section{a. Model}

We consider a single spin- $S$ object, which evolves according to the time-dependent Hamiltonian

$$
H(t)=-h^{x} S^{x}-h^{z} S^{z}-h_{D}^{x} \operatorname{Sgn}(\sin (\omega t)) S^{x} .
$$

Since $\sin (\omega t)$ is positive for $0<t<T / 2$ and negative for $T / 2<t<T$, where $T=2 \pi / \omega$, the Floquet operator is given by

$U=e^{(i T / 2)\left[\left(h^{x}-h_{D}^{x}\right) S^{x}+h^{z} S^{z}\right]} \times e^{(i T / 2)\left[\left(h^{x}+h_{D}^{x}\right) S^{x}+h^{z} S^{z}\right]}$.

The group properties of matrices of the form $e^{i \vec{a} \cdot \vec{S}}$ imply that $U$ in Eq. (B10) must be of the same form and can therefore be written as

$$
\begin{aligned}
U & =e^{i \gamma \hat{k} \cdot \vec{s}}, \\
\text { where } \hat{k} & =(\cos \theta, \sin \theta \cos \phi, \sin \theta \sin \phi)
\end{aligned}
$$

is a unit vector. We work in the basis in which $S^{x}$ is diagonal; hence, we choose the polar angles in such a way that the $x$ component of $\hat{k}$ is equal to $\cos \theta$. The eigenstates of $U$ in Eq. (B11) are the same as the eigenstates of the matrix $M=\hat{k} \cdot \vec{S}$. It is then clear that the expectation values of $S^{x}$ in the different eigenstates take the values $\cos \theta$ times $S, S-1, \ldots,-S$. The maximum expectation value is given by $s_{\max }=S \cos \theta$.

An important point to note is that if the parameters $h^{x}, h^{z}, h_{D}^{x}$, and $T$ are fixed and only the spin $S$ is varied, the values of $\gamma$ and $\hat{k}$ in Eq. (B11) do not change. Thus, if we can calculate these quantities for one particular value of $S$, the results will hold for all $S$. In particular, $m_{\max }^{x} \equiv$ $s_{\max } / S=\cos \theta$ will not depend on $S$. We have confirmed this numerically for a variety of parameter values.

\section{b. Results from FDPT}

Next, we apply the perturbation theory developed in Sec. V. Writing the Hamiltonian as $H=H_{0}(t)+V$, where

$$
\begin{aligned}
H_{0}(t) & =-h^{x} S^{x}-h_{D}^{x} \operatorname{Sgn}(\sin (\omega t)) S^{x}, \\
V & =-h^{z} S^{z},
\end{aligned}
$$

we can use perturbation theory to study how the state given by $|0\rangle \equiv\left|S^{x}=S\right\rangle$ mixes with the state $|1\rangle \equiv\left|S^{x}=S-1\right\rangle$. Following the steps leading up to Eq. (28), and using the fact that $\left\langle 0\left|S^{z}\right| 1\right\rangle=\sqrt{S / 2}$, we find that

$$
c_{1}(0)=\frac{\sqrt{2 S} h^{z}}{h_{D}^{x}} \frac{e^{i h^{x} T / 2}\left[e^{i h_{D}^{x} T / 2}-\cos \left(h^{x} T / 2\right)\right]}{e^{i h^{x} T}-1} .
$$

\section{c. Exact results}

It is instructive to look at the form of the Floquet operator $U$ in different cases. We first derive an exact expression for $U$ using the identity that if

$$
e^{i \alpha \hat{m} \cdot \vec{S}} e^{i \chi \hat{n} \cdot \vec{S}}=e^{i \gamma \hat{k} \cdot \vec{S}}
$$

then

$$
\begin{aligned}
\cos \left(\frac{\gamma}{2}\right)= & \cos \left(\frac{\alpha}{2}\right) \cos \left(\frac{\chi}{2}\right)-\hat{m} \cdot \hat{n} \sin \left(\frac{\alpha}{2}\right) \sin \left(\frac{\chi}{2}\right) \\
\hat{k}= & \frac{1}{\sin (\gamma / 2)}\left[\hat{m} \sin \left(\frac{\alpha}{2}\right) \cos \left(\frac{\chi}{2}\right)\right. \\
& +\hat{n} \sin \left(\frac{\chi}{2}\right) \cos \left(\frac{\alpha}{2}\right) \\
& \left.-\hat{m} \times \hat{n} \sin \left(\frac{\alpha}{2}\right) \sin \left(\frac{\chi}{2}\right)\right]
\end{aligned}
$$


We can derive Eq. (B15) from Eq. (B14) for the case $S=$ $1 / 2$ when $\vec{S}=\vec{\sigma} / 2$. Equation (B15) then follows for any value of $S$ due to the group properties of the matrices given in Eq. (B14).

We now use Eqs. (B14) and (B15) along with Eq. (B11), which can be written in the form

$$
\begin{aligned}
& \alpha=\frac{T}{2} \sqrt{\left(h_{D}^{x}-h^{x}\right)^{2}+\left(h^{z}\right)^{2}}, \\
& \hat{m}=-\frac{\left(h_{D}^{x}-h^{x}\right) \hat{x}-h^{z} \hat{z}}{\sqrt{\left(h_{D}^{x}-h^{x}\right)^{2}+\left(h^{z}\right)^{2}}}, \\
& \chi=\frac{T}{2} \sqrt{\left(h_{D}^{x}+h^{x}\right)^{2}+\left(h^{z}\right)^{2}}, \\
& \hat{n}=\frac{\left(h_{D}^{x}+h^{x}\right) \hat{x}+h^{z} \hat{z}}{\sqrt{\left(h_{D}^{x}-h^{x}\right)^{2}+\left(h^{z}\right)^{2}}},
\end{aligned}
$$

where we have assumed that $h_{D}^{x}$ is positive and much larger than $\left|h^{x}\right|$ and $\left|h^{z}\right|$.

If $e^{i h^{x} T} \neq 1$, we can write the expressions in Eqs. (B16) to zeroth order in the small parameter $h^{z} / h_{D}^{x}$ to obtain

$$
\begin{array}{ll}
\alpha=\frac{T}{2}\left(h_{D}^{x}-h^{x}\right), & \hat{m}=-\hat{x}, \\
\chi=\frac{T}{2}\left(h_{D}^{x}+h^{x}\right), & \hat{n}=\hat{x} .
\end{array}
$$

Equations (B14) and (B15) then imply that

$$
\cos \left(\frac{\gamma}{2}\right)=\cos \left(\frac{h^{x} T}{2}\right), \quad \text { and } \quad \hat{k}=\hat{x} .
$$

We thus find that the Floquet operator for the time period $T$ corresponds to a rotation about the $\hat{x}$ axis.

If $e^{i h^{x} T}=1$, i.e., $\cos \left(h^{x} T / 2\right)= \pm 1$, the denominator of Eq. (B13) vanishes. If $e^{i h_{D}^{x} T / 2} \neq \cos \left(h^{x} T / 2\right)$, we have to expand the expressions in Eqs. (B16) up to second order in $h^{z} / h_{D}^{x}$ to find

$$
\begin{aligned}
\hat{k}= & \cos \left(\frac{h_{D}^{x} T}{4}\right) \hat{z}-\sin \left(\frac{h_{D}^{x} T}{4}\right) \hat{y} \\
& \text { if } \cos \left(\frac{h^{x} T}{2}\right)=1, \\
= & \sin \left(\frac{h_{D}^{x} T}{4}\right) \hat{z}+\cos \left(\frac{h_{D}^{x} T}{4}\right) \hat{y} \\
& \text { if } \cos \left(\frac{h^{x} T}{2}\right)=-1 .
\end{aligned}
$$

Hence, the Floquet operator corresponds to a rotation about an axis lying in the $y-z$ plane, which implies that the expectation value of $S^{x}$ will be zero in all the eigenstates of the Floquet operator.

If $e^{i h^{x} T}=1$ and $e^{i h_{D}^{x} T / 2}=\cos \left(h^{x} T / 2\right)= \pm 1$, both the numerator and denominator of Eq. (B13) vanish. We then discover that

$$
\hat{k}=\frac{h^{x} \hat{x}-h^{z} \hat{z}}{\sqrt{\left(h^{z}\right)^{2}+\left(h^{x}\right)^{2}}} .
$$

In this case, the Floquet operator corresponds to a rotation about an axis lying in the $x-z$ plane.

\section{FDPT for the Ising chain}

The unperturbed energy difference for the Hamiltonian in Eq. (6) is given by

$E_{m}(t)-E_{0}(t)=4(J-\kappa)+2 h_{0}^{x}+2 h_{D}^{x} \operatorname{Sgn}(\sin (\omega t))$.

We now use the notations and results from Sec. V to construct the Floquet state $|\psi(0)\rangle$ obtained by perturbing the unperturbed (Floquet) eigenstate $|0\rangle$ to first order in $V$ given by

$$
\begin{aligned}
\psi(0) & =c_{0}|0\rangle+\sum_{m \neq 0}^{L} c_{m}(0)|m\rangle \\
& =c_{0}|0\rangle+\sqrt{L} c_{m}(0)|L-2\rangle,
\end{aligned}
$$

where

$$
|L-2\rangle \equiv \frac{1}{\sqrt{L}} \sum_{m=1}^{L}|m\rangle
$$

is a translation invariant and normalized state in which $\sum_{m} \sigma_{m}^{x}=L-2$. Taking $c_{0}(t)=1$ for all $t$ and using $\langle m|V| 0\rangle=-h^{z}$ in Eq. (28), we get

$$
c_{m}(0)=i h^{z} \frac{\int_{0}^{T} d t e^{i \int_{0}^{t} d t^{\prime}\left[E_{m}\left(t^{\prime}\right)-E_{0}\left(t^{\prime}\right)\right]}}{e^{i \int_{0}^{T} d t\left[E_{m}(t)-E_{0}(t)\right]}-1},
$$

where $E_{m}(t)-E_{0}(t)$ is given in Eq. (B21).

\section{Failure of FDPT and emergent integrability at the scars}

The FDPT always works well in integrable systems (e.g., the single large-spin case discussed here, and also other examples studied but not shown here). However, FDPT seems to lose accuracy away from integrability and, hence, from the scar points. This is an interesting, indirect indication of the fact that integrability emerges at the scar points. In contrast to the very accurate prediction of resonances in Fig. 4 (main text), Fig. 9 shows a substantial mismatch between the FDPT predictions and the true numerical resonances (dips) away from the scars. 


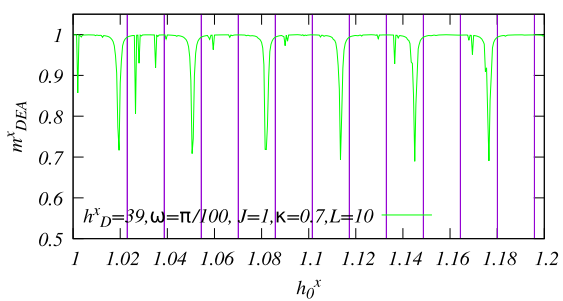

FIG. 9. Mismatches between the numerical dips in $m_{\mathrm{DE}}^{x}$ and the first-order FDPT prediction [vertical lines obtained from Eq. (42)]. The parameters are chosen such that the condition for a scar is not satisfied, i.e., $h_{D}^{x} \neq n \omega$.

\section{APPENDIX C: FREEZE AN ARBITRARY BIT STRING BY TAILORING THE EMERGENT CONSERVATION LAW}

From Fig. 2, we note that the fully polarized state is quite special—at the scar points, its magnetization remains strongly frozen close to unity, and its entanglement entropy does not grow. This is in stark contrast with other $x$-basis states for which, though $m^{x}$ remains conserved, the entanglement entropy experiences substantial growth, which can be understood from the steplike structure (Fig. 1) appearing at the scar points. We expect this phenomenology to be present for other strong drives that divide the Hilbert space into sectors that are, at most, weakly mixed as long as these sectors are separated by finite gaps.

We illustrate this case by arresting the entanglement dynamics of the $L / 2$-domain-pair state, which sees substantial growth of $E_{\frac{1}{2}}$ under the drive with a uniform longitudinal field (Fig. 2, middle column). Instead of a uniform field, we choose the following drive Hamiltonian:

$$
H_{D}=-h_{D}^{x} \sum_{i=1}^{L / 2} \sigma_{i}^{x}+h_{D}^{x} \sum_{i=L / 2+1}^{L} \sigma_{i}^{x}
$$

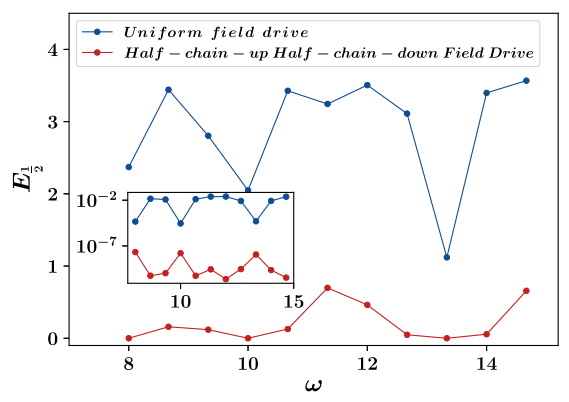

FIG. 10. Freezing of the entanglement growth of the $L / 2$ domain-pair $x$-basis state under a half-up half-down field drive [Eq. (C1)]. The fate of a fully polarized state under the same drive is also shown for comparison. The main frame shows $E_{\frac{1}{2}}$, while the inset shows $\mathrm{m}^{x}$, averaged over $10^{4}$ cycles, after driving for $10^{10}$ cycles. The results are for $J=1, \kappa=0.7$, $h_{0}^{x}=e / 10, h_{D}^{x}=40, L=14$. keeping the rest of the setup the same as given by Eq. (6). For $H_{D}$ of the above form, the $L / 2$-domain-pair state is in an eigensector of its own. As expected, the entanglement growth is strongly suppressed for the $L / 2$-domain-pair state-especially at $\omega=8,10$, and 13.33 , which are the scar points corresponding to the applied drive amplitude $h_{D}^{x}=40$ - while a substantial growth of entanglement is observed for the fully polarized initial state (see Fig. 10). This is in stark contrast to the results for the uniform drive (left and middle columns of Fig. 2).

[1] M. Srednicki, Chaos and Quantum Thermalization, Phys. Rev. E 50, 888 (1994).

[2] M. Rigol, V. Dunjko, and M. Olshanii, Thermalization and its Mechanism for Generic Isolated Quantum Systems, Nature (London) 452, 854 (2008).

[3] A. Lazarides, A. Das, and R. Moessner, Equilibrium States of Generic Quantum Systems Subject to Periodic Driving, Phys. Rev. E 90, 012110 (2014).

[4] L. D'Alessio and M. Rigol, Long-Time Behavior of Isolated Periodically Driven Interacting Lattice Systems, Phys. Rev. X 4, 041048 (2014).

[5] D. M. Basko, I. L. Aleiner, and B. L. Altshuler, MetalInsulator Transition in a Weakly Interacting Many-Electron System with Localized Single-Particle States, Ann. Phys. (N.Y.) 321, 1126 (2006).

[6] Special Issue: Many-Body Localization, Vol. 529, edited by J. H. Bardarson, Frank Pollmann, H. Schneider, and S. L. Sondhi Annelen der Physik (2017).

[7] S. Sachdev, K. Sengupta, and S. M. Girvin, Mott Insulators in Strong Electric Fields, Phys. Rev. B 66, 075128 (2002).

[8] E. van Nieuwenburg, Y. Baum, and G. Refael, From Bloch Oscillations to Many-Body Localization in Clean Interacting Systems, Proc. Natl. Acad. Sci. U.S.A. 116, 9269 (2019).

[9] M. Schulz, C. A. Hooley, R. Moessner, and F. Pollmann, Stark Many-Body Localization, Phys. Rev. Lett. 122, 040606 (2019).

[10] A. Lazarides, A. Das, and R. Moessner, Fate of Many-Body Localization under Periodic Driving, Phys. Rev. Lett. 115, 030402 (2015).

[11] P. Ponte, Z. Papić, F. Huveneers, and D. A. Abanin, ManyBody Localization in Periodically Driven Systems, Phys. Rev. Lett. 114, 140401 (2015).

[12] B. Mukherjee, S. Nandy, A. Sen, D. Sen, and K. Sengupta, Collapse and Revival of Quantum Many-Body Scars via Floquet Engineering, Phys. Rev. B 101, 245107 (2020).

[13] C. W. von Keyserlingk, V. Khemani, and S. L. Sondhi, Absolute Stability and Spatiotemporal Long-Range Order in Floquet Systems, Phys. Rev. B 94, 085112 (2016).

[14] D. V. Else, B. Bauer, and C. Nayak, Floquet Time Crystals, Phys. Rev. Lett. 117, 090402 (2016).

[15] H. Bernien, S. Schwartz, A. Keesling, H. Levine, A. Omran, H. Pichler, S. Choi, A. S. Zibrov, M. Endres, M. Greiner, V. Vuletic, and M. D. Lukin, Probing Many-Body Dynamics on a 51-Atom Quantum Simulator, Nature (London) 551, 579 (2017). 
[16] C. J. Turner, A. A. Michailidis, D. A. Abanin, M. Serbyn, and Z. Papic, Weak Ergodicity Breaking from Quantum Many-Body Scars, Nat. Phys. 14, 745 (2018).

[17] N. Shiraishi and T. Mori, Systematic Construction of Counterexamples to the Eigenstate Thermalization Hypothesis, Phys. Rev. Lett. 119, 030601 (2017).

[18] C. J. Turner, A. A. Michailidis, D. A. Abanin, M. Serbyn, and Z. Papić, Quantum Scarred Eigenstates in a Rydberg Atom Chain: Entanglement, Breakdown of Thermalization, and Stability to Perturbations, Phys. Rev. B 98, 155134 (2018).

[19] V. Khemani, C. R. Laumann, and A. Chandran, Signatures of Integrability in the Dynamics of Rydberg-Blockaded Chains, Phys. Rev. B 99, 161101(R) (2019).

[20] W. W.I Ho, S. Choi, H. Pichler, and M. D. Lukin, Periodic Orbits, Entanglement, and Quantum Many-Body Scars in Constrained Models: Matrix Product State Approach, Phys. Rev. Lett. 122, 040603 (2019).

[21] S. Choi, C. J. Turner, H. Pichler, W. W. Ho, A. A. Michailidis, Z. Papić, M. Serbyn, M. D. Lukin, and D. A. Abanin, Emergent SU(2) Dynamics and Perfect Quantum Many-Body Scars, Phys. Rev. Lett. 122, 220603 (2019).

[22] S. Moudgalya, N. Regnault, and B. A. Bernevig, Entanglement of Exact Excited States of Affleck-KennedyLieb-Tasaki Models: Exact Results, Many-Body Scars, and Violation of the Strong Eigenstate Thermalization Hypothesis, Phys. Rev. B 98, 235156 (2018).

[23] V. Khemani, M. Hermele, and R. Nandkishore, Localization from Hilbert Space Shattering: From Theory to Physical Realizations, Phys. Rev. B 101, 174204 (2020).

[24] S. Moudgalya, S. Rachel, B. A. Bernevig, and N. Regnault, Exact Excited States of Nonintegrable Models, Phys. Rev. B 98, 235155 (2018).

[25] R. Mondaini, K. Mallayya, L. F. Santos, and M. Rigol, Comment on Systematic Construction of Counterexamples to the Eigenstate Thermalization Hypothesis, Phys. Rev. Lett. 121, 038901 (2018).

[26] L. D'Alessio and A. Polkovnikov, Many-Body Energy Localization Transition in Periodically Driven Systems, Ann. Phys. (N.Y.) 333, 19 (2013).

[27] M. Bukov, M. Heyl, D. A. Huse, and A. Polkovnikov, Heating and Many-Body Resonances in a Periodically Driven Two-Band System, Phys. Rev. B 93, 155132 (2016).

[28] M. Bukov, L. D’Alessio, and A. Polkovnikov, Universal High-Frequency Behavior of Periodically Driven Systems: From Dynamical Stabilization to Floquet Engineering, Adv. Phys. 64, 139 (2015).

[29] A. Agarwala and D. Sen, Effects of Interactions on Periodically Driven Dynamically Localized Systems, Phys. Rev. B 95, 014305 (2017).

[30] S. Dasgupta, U. Bhattacharya, and A. Dutta, Phase Transition in the Periodically Pulsed Dicke Model, Phys. Rev. E 91, 052129 (2015).

[31] P. Bordia, H. Lüschen, U. Schneider, M. Knap, and I. Bloch, Thermalization and Its Mechanism for Generic Isolated Quantum Systems, Nat. Phys. 13, 460 (2017).

[32] S. Pal, N. Nishad, T. S. Mahesh, and G. J. Sreejith, Temporal Order in Periodically Driven Spins in Star-Shaped Clusters, Phys. Rev. Lett. 120, 180602 (2018).
[33] T. Qin and W. Hofstetter, Nonequilibrium Steady States and Resonant Tunneling in Time-Periodically Driven Systems with Interactions, Phys. Rev. B 97, 125115 (2018).

[34] K. Seetharam, P. Titum, M. Kolodrubetz, and G. Refael, Absence of Thermalization in Finite Isolated Interacting Floquet Systems, Phys. Rev. B 97, 014311 (2018).

[35] T. Prosen, Time Evolution of a Quantum Many-Body System: Transition from Integrability to Ergodicity in the Thermodynamic Limit, Phys. Rev. Lett. 80, 1808 (1998).

[36] T. Prosen, General Relation between Quantum Ergodicity and Fidelity of Quantum Dynamics, Phys. Rev. E 65, 036208 (2002).

[37] D. J. Luitz, A. Lazarides, and Y. B. Lev, Periodic and Quasiperiodic Revivals in Periodically Driven Interacting Quantum Systems, Phys. Rev. B 97, 020303(R) (2018).

[38] A. Haldar, R. Moessner, and A. Das, Onset of Floquet Thermalization, Phys. Rev. B 97, 245122 (2018).

[39] A. Das, Exotic Freezing of Response in a Quantum ManyBody System, Phys. Rev. B 82, 172402 (2010).

[40] S. Bhattacharyya, A. Das, and S. Dasgupta, Transverse Ising Chain under Periodic Instantaneous Quenches: Dynamical Many-Body Freezing and Emergence of Slow Solitary Oscillations, Phys. Rev. B 86, 054410 (2012).

[41] S. S. Hegde, H. Katiyar, T. S. Mahesh, and A. Das, Freezing a Quantum Magnet by Repeated Quantum Interference: An Experimental Realization, Phys. Rev. B 90, 174407 (2014).

[42] S. Mondal, D. Pekker, and K. Sengupta, Dynamics-Induced Freezing of Strongly Correlated Ultracold Bosons, Europhys. Lett. 100, 60007 (2012).

[43] A. Russomanno, A. Silva, and G. E. Santoro, Linear Response as a Singular Limit for a Periodically Driven Closed Quantum System, J. Stat. Mech. (2013) P09012.

[44] A. Lazarides, A Das, and R Moessner, Periodic Thermodynamics of Isolated Systems, Phys. Rev. Lett. 112, 150401 (2014).

[45] K. Seetharam, P. Titum, M. Kolodrubetz, and G. Refael, Absence of Thermalization in Finite Isolated Interacting Floquet Systems, Phys. Rev. B 97, 014311 (2018).

[46] M. Rodriguez-Vega, M. Lentz, and B. Seradjeh, Floquet Perturbation Theory: Formalism and Application to LowFrequency Limit, New J. Phys. 20, 093022 (2018).

[47] M. Vogl, P. Laurell, A. D. Barr, and G. A. Fiete, Flow Equation Approach to Periodically Driven Quantum Systems, Phys. Rev. X 9, 021037 (2019).

[48] M. Vogl, M. Rodriguez-Vega, and G. A. Fiete, Effective Floquet Hamiltonian in the Low-Frequency Regime, Phys. Rev. B 101, 024303 (2020).

[49] M. Srednicki, Does Quantum Chaos Explain Quantum Statistical Mechanics?, arXiv:cond-mat/9410046v2.

[50] M. Rigol, V. Dunjko, V. Yurovsky, and M. Olshanii, Relaxation in a Completely Integrable Many-Body Quantum System: An Ab Initio Study of the Dynamics of the Highly Excited States of 1D Lattice Hard-Core Bosons, Phys. Rev. Lett. 98, 050405 (2007).

[51] A. C. Cassidy, C. W. Clark, and M. Rigol, Generalized Thermalization in an Integrable Lattice System, Phys. Rev. Lett. 106, 140405 (2011).

[52] P. Reimann, Foundation of Statistical Mechanics under Experimentally Realistic Conditions, Phys. Rev. Lett. 101, 190403 (2008). 
[53] A. Eckardt and E. Anisimovas, High-Frequency Approximation for Periodically Driven Quantum Systems from a Floquet-Space Perspective, New J. Phys. 17, 093039 (2015).

[54] A. Soori and D. Sen, Nonadiabatic Charge Pumping by Oscillating Potentials in One Dimension: Results for Infinite System and Finite Ring, Phys. Rev. B 82, 115432 (2010).

[55] J. Zhang, P. W. Hess, A. Kuprianidis, P. Becker, A. Lee, J. Smith, G. Pagano, I.-D. Potirniche, A. C. Potter, A. Vishwanath, N. Y. Yao, and C Monroe, Observation of a Discrete Time Crystal, Nature (London) 543, 217 (2017).

[56] J. Rovny, R. L. Blum, and S. E. Barrett, Observation of Discrete-Time-Crystal Signatures in an Ordered Dipolar Many-Body System, Phys. Rev. Lett. 120, 180603 (2018).
[57] A. Das and R. Moessner, Switching the Anomalous DC Response of an AC-driven Quantum Many-Body System, arXiv:1208.0217.

[58] M. Bukov, Ph.D. thesis, Boston University, 2016, https:// mgbukov.github.io/files/Floquet_thesis.pdf.

[59] P. Weinberg and M. Bukov, QuSpin: A Python Package for Dynamics and Exact Diagonalisation of Quantum Many Body Systems Part I: Spin Chains, SciPost Phys. 2, 003 (2017).

[60] P. Weinberg and M.Bukov, QuSpin: A Python Package for Dynamics and Exact Diagonalisation of Quantum Many Body Systems. Part II: Bosons, Fermions and Higher Spins, SciPost Phys. 7, 20 (2019).

[61] D. J. Luitz, R. Moessner, S. L. Sondhi, and V. Khemani, Prethermalization without Temperature, Phys. Rev. X 10, 021046 (2020). 